\title{
Lesotho: Statistical Annex
}

This Statistical Annex on Lesotho was prepared by a staff team of the International Monetary Fund as background documentation for the periodic consultation with the member country. It is based on the information available at the time it was completed on February 14, 2001. The views expressed in this document are those of the staff team and do not necessarily reflect the views of the government of Lesotho or the Executive Board of the IMF.

The policy of publication of staff reports and other documents by the IMF allows for the delction of market-sensitive information.

To assist the IMF in evaluating the publication policy, reader comments are invited and may be sent by e-mail to Publicationpolicy@imf.org.

Copies of this report are available to the public from International Monetary Fund - Publication Services 700 19th Street, N.W. - Washington, D.C. 20431

Telephone: (202) 6237430 • Telefax: (202) 6237201

E-mail: publications@imf.org • Internet: http://www.imf.org

Price: $\$ 15.00$ a copy

International Monetary Fund

Washington, D.C. 


\section{INTERNATIONAL MONETARY FUND}

\section{LESOTHO}

\section{Statistical Annex}

Prepared by a staff mission consisting of Mr. P. Ramlogan (head), Mr. P. Lopes; Ms. S. Medina-Cas,

Mr. E. Flores and Mr. J. Sebti (all-AFR)

Approved by the African Department

February 14, 2001

\section{Contents}

Basic Data . 3

Statistical Tables

National Accounts, Prices, and Employment

1. Gross Domestic Product by Sector (at constant 1995 prices), 1995/96-1999/2000.

2. Gross Domestic Product by Sector (at current prices), 1995/96-1999/2000. 5

3. Gross Domestic Product by Expenditure, 1995/96-1999/2000 …........................ 6

4. Consumer Price Indices, January 1996-October 2000 ….............................. 7

5. Basic Monthly Minimum Wages, 1995-99 …................................................ 8

6. Public Service Employment, 1995/96-1999/2000 ….................................... 9

Public Finance

7. Central Government Operations, 1995/96-1999/2000 .................................... 10

8. Government Revenue and Grants, 1995/96-1999/2000 ................................ 11

9. Southern African Customs Union (SACU) Operations, 1995/96-1999/2000 … 12

10. Economic Classification of Government Expenditure, 1995/96-1999/2000 ...... 13

11. Functional Classification of Government Expenditure, 1995/96-1999/2000 ..... 14

12. Outstanding Government Domestic Debt by Instrument and Holder, $1995-99$ 


\section{Money and Banking}

13. Monetary Survey, March 1995-September 2000 .

14. Assets and Liabilities of the Central Bank of Lesotho, March 1995-September 2000

15. Assets and Liabilities of Commercial Banks, March 1995-September 2000 _..... 18

16. Principal Aggregates of Commercial Banks' Operations, March 1994-September 2000

17. Sectoral Distribution of Commercial Bank Credit to the Private Sector and Statutory Bodies, March 1995-September 2000

18. Interest Rates Paid by the Central Bank on Commercial Bank Deposits, March 1994-September 2000...

19. Interest Rates at Commercial Banks, March 1995-September 2000

20. Comparative Money Market Rates, March 1994-September 2000

\section{External Sector}

21. Balance of Payments, 1995/96-1999/2000 (In millions of U.S. dollars) ........... 24

22. Balance of Payments, 1995/96-1999/2000 (In millions of maloti) ................... 25

23. Services and Income Account, 1995/96-1999/2000 …................................. 26

24. Lesotho Miners in South Africa, 1995-99 ……....................................... 27

25. Composition of Recorded Exports, 1995-99 …........................................... 28

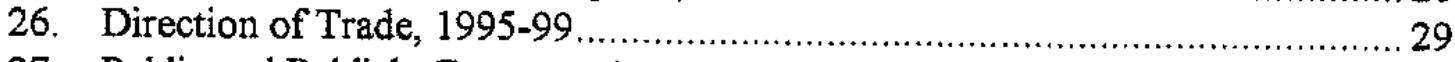

27. Public and Publicly Guaranteed External Debt Outstanding, 1995/96-1999/2000

Appendices

1. Summary of the Tax System

2. Summary of the Exchange and Trade System 
Lesotho: Basic Data 1/

Area, population, and income

Area (thousand sq. $\mathrm{km}$ )

Population (1998; in millions)

Annual demographic growth rate (1992.98; in percent)

GNP per capita (1998; in U.S. dollers)

30,355

2.1

696

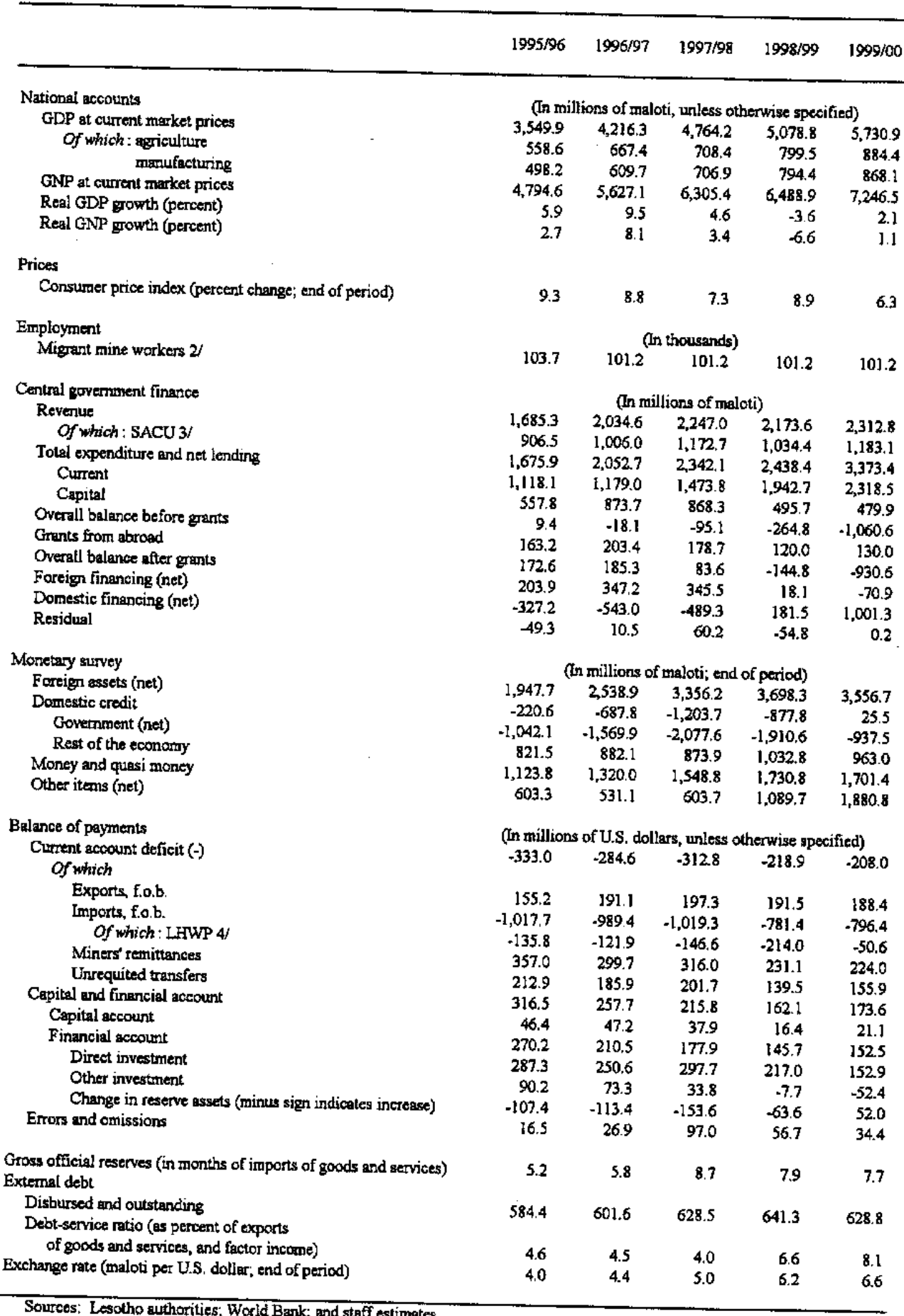

Sources: Lesotho althorities; World Bank; and staff estimetes.

1/ Fiscal year is April-March

2/ Average number employed in South Africs; data refer to calendar years,

3/ Southem Africen Customs Union

4/ Lesotho Highlands Water Project. 
Table 1. Lesotho: Gross Domestic Product by Sector (at constant 1995 prices), 1995/96-1999/2000 1/2/ (In millions of maloti)

\begin{tabular}{|c|c|c|c|c|c|}
\hline & $1995 / 96$ & $1996 / 97$ & $1997 / 98$ & $1998 / 99$ & $1999 / 00$ \\
\hline Primary sector & 547.7 & 618.1 & 619.6 & 624.0 & 640.7 \\
\hline Crops & 323.4 & 414.8 & 399.9 & 383.9 & 375.8 \\
\hline Livestock & 190.5 & 169.9 & 184.3 & 205.9 & 232.8 \\
\hline Agricultural services & 33.8 & 33.4 & 35.4 & 34.2 & 32.1 \\
\hline Secondary sector & $1,182.8$ & $1,312.7$ & $1,421.9$ & $1,311.3$ & $1,385.4$ \\
\hline Mining and quarrying & 3.2 & 3.1 & 3.3 & 2.6 & 2.7 \\
\hline Manufacturing and handicraft & 485.8 & 541.5 & 555.9 & 540.0 & 556.5 \\
\hline Electricity and water & 85.1 & 130.9 & 225.1 & 179.0 & 212.5 \\
\hline Construction & 317.4 & 297.9 & 342.1 & 414.2 & 484.9 \\
\hline LHWP construction $3 /$ & 291.4 & 339.4 & 295.4 & 175.5 & 128.8 \\
\hline Tertiary sector & $1,283.9$ & $1,366.0$ & $1,432.9$ & $1,443.7$ & $1,457.1$ \\
\hline Wholesale and retail & 280.0 & 304.2 & 319.7 & 305.6 & 294.7 \\
\hline Hotel and restaurants & 41.8 & 47.4 & 47.8 & 43.6 & 44.1 \\
\hline Transport and storage & 72.5 & 75.5 & 76.7 & 71.5 & 69.7 \\
\hline Post and telecommunications & 46.4 & 50.5 & 59.2 & 59.6 & 63.0 \\
\hline Financial intermediation & 113.7 & 113.2 & 103.1 & 112.0 & 141.5 \\
\hline Owner-occupied dwellings & 153.6 & 156.6 & 159.8 & 163.0 & 166.4 \\
\hline Other real estate and business services & 59.5 & 64.4 & 77.4 & 67.7 & 61.6 \\
\hline Public administration & 235.7 & 247.5 & 261.6 & 286.0 & 294.9 \\
\hline Education & 275.9 & 285.7 & 300.8 & 308.6 & 313.6 \\
\hline Health and social work & 58.1 & 62.4 & 60.9 & 66.1 & 69.2 \\
\hline Community, social, and personal services & 40.3 & 40.6 & 41.1 & 41.7 & 42.3 \\
\hline Less: financial services indirectly measured & -93.4 & -82.0 & -75.1 & -81.3 & -104.0 \\
\hline GDP at producers' prices & $3,014.3$ & $3,296.8$ & $3,474.4$ & $3,379.0$ & $3,483.2$ \\
\hline Plus: taxes on products & 453.6 & 499.7 & 495.6 & 448.5 & 424.8 \\
\hline GDP at purchasers' prices & $3,467.9$ & $3,796.4$ & $3,970.0$ & $3,827.5$ & $3,908.0$ \\
\hline Plus: net factor income from abroad & $1,217.1$ & $1,269.2$ & $1,266.3$ & $1,062.6$ & $1,033.5^{-}$ \\
\hline Gross national income at purchasers' prices & $4,685.1$ & $5,065.7$ & $5,236.3$ & $4,890.2$ & $4,941.5$ \\
\hline
\end{tabular}

Sources: Lesotho Bureau of Statistics; Central Bank of Lesotho; Lesotho Highlands Development Authority; and staff estimates.

1/ Fiscal year begins April 1.

2/ Fiscal year estimates based on sectoral calendar-year data compiled by the Bureau of Statistics.

3/ Lesotho Highlands Water Project (LHWP). 
Table 2. Lesotho: Gross Domestic Product by Sector (at current prices), 1995/96-1999/2000 1/2/

(In millions of maloti)

\begin{tabular}{|c|c|c|c|c|c|}
\hline & $1995 / 96$ & $1996 / 97$ & $1997 / 98$ & $1998 / 99$ & $1999 / 00$ \\
\hline Primary sector & 558.6 & 667.4 & 708.4 & 799.5 & 884.4 \\
\hline Crops & 333.6 & 454.7 & 454.4 & 495.5 & 515.4 \\
\hline Livestock & 190.4 & 174.2 & 207.3 & 253.4 & 316.3 \\
\hline Agricultural services & 34.6 & 38.5 & 46.8 & 50.6 & 52.7 \\
\hline Secondary sector & $1,217.9$ & $1,497.3$ & $1,766.3$ & $1,810.9$ & $2,131.5$ \\
\hline Mining and quarrying & 3.2 & 3.1 & 3.7 & 4.1 & 5.0 \\
\hline Manufacturing and handicraft & 498.2 & 609.7 & 706.9 & 794.4 & 868.1 \\
\hline Electricity and water & 92.7 & 171.2 & 291.6 & 245.2 & 309.5 \\
\hline Construction & 319.6 & 315.0 & 386.8 & 514.0 & 743.9 \\
\hline LHWP construction 3/ & 304.1 & 398.2 & 377.3 & 253.2 & 205.1 \\
\hline Tertiary sector & $1,317.9$ & $1,543,4$ & $1,775.3$ & $1,948.7$ & $2,113.8$ \\
\hline Wholesale and retail & 286.9 & 342.8 & 397.7 & 412.4 & 434.1 \\
\hline Hotel and restaurants & 42.9 & 53.0 & 58.1 & 57.4 & 61.9 \\
\hline Transport and storage & 76.8 & 96.4 & 107.8 & 104.4 & 105.6 \\
\hline Post and telecommunications & 46.1 & 48.6 & 55.3 & 59.1 & 67.9 \\
\hline Financial intermediation & 116.5 & 127.3 & 127.3 & 142.5 & 183.4 \\
\hline Owner-occupied dwellings & 156.7 & 170.1 & 177.8 & 187.2 & 196.4 \\
\hline Other real estate and business services & 59.5 & 68.5 & 94.3 & 87.3 & 84.5 \\
\hline Public administration & 242.1 & 284.8 & 346.6 & 425.2 & 484.3 \\
\hline Education & 285.3 & 329.8 & 377.4 & 434.1 & 473.0 \\
\hline Health and social work & 59.4 & 69.9 & 77.2 & 90.9 & 100.8 \\
\hline Community, social, and personal services & 41.0 & 44.9 & 50.0 & 54.6 & 59.1 \\
\hline Less: financial services indirectly measured & -95.5 & -92.7 & -94.3 & -106.4 & -137.1 \\
\hline GDP at producers' prices & $3,094.4$ & $3,708.1$ & $4,250.0$ & $4,559.1$ & $5,129.8$ \\
\hline Plus: taxes on products & 455.5 & 508.2 & 514.3 & 519.7 & 601.2 \\
\hline GDP at purchasers' prices & $3,549.9$ & $4,216.3$ & $4,764.2$ & $5,078.8$ & $5,730.9$ \\
\hline Plus: net factor income from abroad & $1,244.7$ & $1,410.9$ & $1,541.2$ & $1,410.1$ & $1,515.6$ \\
\hline Gross national income at purchasers' prices & $4,794.6$ & $5,627.1$ & $6,305.4$ & $6,488.9$ & $7,246.5$ \\
\hline
\end{tabular}

Sources: Lesotho Bureau of Statistics; Central Bank of Lesotho; Lesotho Highlands Development Authority; and staff estimates.

I/ Fiscal year begins April I.

2/ Fiscal year estimates based on sectoral calendar-year data compiled by the Bureau of Statistics.

3/ Lesotho Highlands Water Project (LHWP). 
Table 3. Lesotho: Gross Domestic Product by Expenditure, 1995/96-1999/2000 1/2/

\begin{tabular}{|c|c|c|c|c|c|}
\hline . & $1995 / 96$ & $1996 / 97$ & $1997 / 98$ & $1998 / 99$ & $1999 / 00$ \\
\hline & \multicolumn{5}{|c|}{ (In millions of maloti) } \\
\hline Gross domestic product & $3,549.9$ & $4,216.3$ & $4,764.2$ & $5,078.8$ & $5,730.9$ \\
\hline Net factor income from abroad & $1,244.7$ & $1,410.9$ & $1,541.2$ & $1,410.1$ & $1,515.6$ \\
\hline Gross national product & $4,794.6$ & $5,627.1$ & $6,305.4$ & $6,488.9$ & $7,246.5$ \\
\hline Unrequited transfers & 783.2 & 833.5 & 951.6 & 811.0 & 961.2 \\
\hline Gross national disposabie income 3 / & $5,577.7$ & $6,460.6$ & $7,257.0$ & $7,299.9$ & $8,207.7$ \\
\hline Consumption & $4,675.6$ & $5,324.1$ & $6,243.5$ & $6,185.1$ & $6,977.7$ \\
\hline Government consumption & $1,058.9$ & $1,112.7$ & $1,383.8$ & $1,814.2$ & $2,135.1$ \\
\hline Private consumption & $3,616.7$ & $4,211.4$ & $4,859.7$ & $4,370.9$ & $4,842.6$ \\
\hline Gross national savings 4/ & 902.2 & $1,136.5$ & $1,013.4$ & $1,114.8$ & $1,230.0$ \\
\hline Public savings 5/ & 626.4 & 921.9 & 863.2 & 359.4 & 177.7 \\
\hline Private savings $6 /$ & 275.8 & 214.6 & 150.2 & 755.4 & $1,052.3$ \\
\hline Investment & $2,127.2$ & $2,412.6$ & $2,489.2$ & $2,386.8$ & $2,511.9$ \\
\hline Gross fixed capital formation & $2,143.5$ & $2,418.5$ & $2,547.9$ & $2,471.0$ & $2,554.5$ \\
\hline Government & 557.8 & 873.7 & 868.3 & 495.7 & 479.9 \\
\hline Private & 565.7 & 428.8 & 195.2 & $1,052.8$ & $1,378.8$ \\
\hline LHWP 7/ & $1,020.0$ & $1,116.0$ & $1,484.4$ & 922.5 & 695.8 \\
\hline Change in stocks & -16.3 & -5.9 & -58.7 & -84.3 & -42.6 \\
\hline \multirow[t]{2}{*}{ Gross national savings less investment 8 / } & $-1,225.1$ & $-1,276.1$ & $-1,475.8$ & $-1,272.0$ & $-1,281.9$ \\
\hline & \multicolumn{5}{|c|}{ (In percent of GDP) } \\
\hline Gross domestic product & 100.0 & 100.0 & 100.0 & 100.0 & 100.0 \\
\hline Net factor income from abroad & 35.1 & 33.5 & 32.3 & 27.8 & 26.4 \\
\hline Gross national product & 135.1 & 133.5 & 132.3 & 127.8 & 126.4 \\
\hline Unrequited transfers & 22.1 & 19.8 & 20.0 & 16.0 & 16.8 \\
\hline Gross national disposable income $3 /$ & 157.1 & 153.2 & 152.3 & 143.7 & 143.2 \\
\hline Consumption & 131.7 & 126.3 & 131.1 & 121.8 & 121.8 \\
\hline Government consumption & 29.8 & 26.4 & 29.0 & 35.7 & 37.3 \\
\hline Private consumption & 101.9 & 99.9 & 102.0 & 86.1 & 84.5 \\
\hline Gross national savings 4/ & 25.4 & 27.0 & 21.3 & 21.9 & 21.5 \\
\hline Public savings $5 /$ & 17.6 & 21.9 & 18.1 & 7.1 & 3.1 \\
\hline Private savings 6 & 7.8 & 5.1 & 3.2 & 14.9 & 18.4 \\
\hline Investment & 59.9 & 57.2 & 52.2 & 47.0 & 43.8 \\
\hline Gross fixed capital formation & 60.4 & 57.4 & 53.5 & 48.7 & 44.6 \\
\hline Government & 15.7 & 20.7 & 18.2 & 9.8 & 8.4 \\
\hline Private & 15.9 & 10.2 & 4.1 & 20.7 & 24.1 \\
\hline LHWP $7 /$ & 28.7 & 26.5 & 31.2 & 18.2 & 12.1 \\
\hline Change in stocks & -0.5 & -0.1 & -1.2 & -1.7 & -0.7 \\
\hline Gross national savings less investment 8 / & -34.5 & .30 .3 & -31.0 & -25.0 & -22.4 \\
\hline
\end{tabular}

Sources: Lesotho authorities; and staff estimates.

1/ Fiscal year beginning in April.

2/ Fiscal year estimates based on calendar-year estimates compiled by the Bureau of Statistics.

3/ Gross national product plus unrequited transfers.

4/ Gross national disposable income less consumption.

5/ Government revenues plus grants less government current expenditures (excluding interest payments).
6/ Estimated as a residual.

7/ Lesotho Highlands Water Project (LHWP).

8/ Equivalent to the external current account balance. 
Table 4. Lesotho: Consuner Price Indices, January 1996 - October 2000 (April 1997 - 100, unless otherwise indicated)

\begin{tabular}{|c|c|c|c|c|c|c|c|c|c|c|c|c|c|c|c|c|c|c|c|c|c|}
\hline & \multirow[b]{2}{*}{ Weights } & \multicolumn{4}{|c|}{1996} & \multicolumn{4}{|c|}{1997} & \multicolumn{4}{|c|}{1998} & \multicolumn{4}{|c|}{1999} & \multicolumn{4}{|c|}{2000} \\
\hline & & Jan. & Apr. & July & Oct. & Jan. & Apr. & Juby & Oct. & Jan. & Apr. & July & $\overrightarrow{\text { Oet }}$ & Jan. & Apr. & July & Oet. & Jan. & Apr. & July & Oct \\
\hline Consumer price index (CPl) 1 & 100.0 & 89.5 & 91.9 & 93.7 & 95.6 & 97.2 & 100.0 & 102.1 & 103.1 & 104.9 & 107.3 & 109.1 & 112.5 & 115.1 & 116.9 & 119.2 & 120.1 & 122.3 & 124.3 & 125.8 & 127.3 \\
\hline Food, beverages, and tobrace & 43.1 & 88.1 & 91.3 & 92.7 & 94.6 & 96.2 & 100.0 & 102.3 & 102.8 & 104.5 & 108.2 & 110.5 & 114.1 & 115.7 & 117.2 & 119.9 & 120.0 & 122.3 & 124.8 & 125.8 & 125.6 \\
\hline $\begin{array}{l}\text { Bread and cereals } \\
\text { Meat and meat products }\end{array}$ & 19.3 & 88.7 & $\begin{array}{l}92.0 \\
91.9\end{array}$ & $\begin{array}{l}93.6 \\
92.9\end{array}$ & 95.5 & 97.0 & 100.0 & 101.0 & 101.2 & 101.1 & 105.1 & 107.0 & 109.4 & 110.4 & 110.6 & 114.0 & 113.5 & 116.0 & 116.8 & $\$ 16.6$ & 115.3 \\
\hline $\begin{array}{l}\text { Meat and meat products } \\
\text { Fnits and vegetables }\end{array}$ & $\begin{array}{l}4.1 \\
4.0\end{array}$ & $\begin{array}{l}92.0 \\
89.8\end{array}$ & $\begin{array}{l}91.9 \\
91.3\end{array}$ & $\begin{array}{l}92.9 \\
91.3\end{array}$ & 94.8 & 96.3 & 100.0 & 99.2 & 102.2 & 107.1 & 109.5 & 111.0 & 113.2 & 115.3 & 115.3 & 116.3 & 118.0 & 119.8 & 120.0 & 121.1 & 121.3 \\
\hline $\begin{array}{l}\text { Fnits and vegetables } \\
\text { Alcoholic beverages and tobacco }\end{array}$ & 8.1 & 82.2 & 89.5 & $\begin{array}{l}91.3 \\
90.0\end{array}$ & 93.2 & 94.7 & 100.0 & 105.0 & 107.2 & 114.2 & 119.7 & 123.8 & 129.8 & 136.6 & 138.5 & 142.6 & 144.7 & 149.8 & 151.5 & 155.1 & 160.7 \\
\hline $\begin{array}{l}\text { Alcohotic beverages and tobacco } \\
\text { Food and thinks }\end{array}$ & 7.6 & 89.6 & 92.8 & $\begin{array}{l}90.0 \\
94.2\end{array}$ & $\begin{array}{l}91.8 \\
96.1\end{array}$ & 93.3 & 100.0 & 101.9 & 102.0 & 103.6 & $\begin{array}{l}107.5 \\
109.9\end{array}$ & $\begin{array}{l}109.9 \\
112.5\end{array}$ & $\begin{array}{l}114.6 \\
118.0\end{array}$ & 114.9 & 120.6 & 123.7 & 124.4 & 125.9 & 133.7 & 134.8 & 136.4 \\
\hline Food and dinks & & & & & 96.1 & 97.7 & 100.0 & 102.3 & 105.9 & 107.3 & 109.9 & 112.5 & 118.0 & 119.2 & & & & & & & \\
\hline Clothing and footwear & 15.8 & 91.5 & 93.0 & 95.7 & 97.7 & 99.3 & 100.0 & 102.2 & 104.7 & 106.8 & 108.9 & 110.7 & 114.9 & 118.2 & 120.8 & 121.7 & 122.8 & 124.7 & 125.4 & 126.4 & 129.1 \\
\hline Blankets & 3.3 & 91.6 & 93.5 & 98.1 & 100.1 & 101.8 & 100.0 & 102.4 & 105.0 & 105.0 & 107.3 & 109.7 & 111.5 & 114.2 & 114.4 & 113.6 & 113.6 & 112.9 & 114.3 & 117.1 & 123.3 \\
\hline Other clothing & 6.3 & 90.7 & 92.0 & 94.1 & 96.0 & 97.5 & 100.0 & 102.3 & 104.4 & 106.3 & 109.3 & 110.9 & 312.7 & 116.1 & 119.9 & 122.3 & 122.3 & 323.6 & 123.9 & 125.1 & 127.2 \\
\hline Footwear & 6.2 & 92.7 & 94.3 & 97.2 & 99.1 & 100.8 & 100.0 & 101.8 & 105.9 & 108.2 & 109.3 & 111.0 & 119.1 & 122.0 & 124.6 & 125.1 & 128.3 & 131.4 & 132.1 & 132.1 & 133.7 \\
\hline Gross rent, fuel, and power $2 f$ & 3.9 & 81.4 & $\mathbf{B B . 9}$ & 92.3 & 94.2 & 95.7 & 100,0 & 100.3 & 101.2 & 104.1 & 104.5 & 107.2 & 109.5 & 110.3 & 109.7 & 109.8 & 110.1 & 312.3 & 113.4 & 115.9 & 120.6 \\
\hline Gross nent and water charges $2 /$ & 0.6 & 100.2 & 100.0 & 100.0 & 102.0 & 103.7 & 100.0 & 100.0 & 100.0 & 100.0 & 100.0 & 100.0 & 100.0 & 100.0 & 100.0 & 100.0 & 100.0 & 100,0 & 101.5 & 101.5 & 101.5 \\
\hline Fuel and power & 3.3 & 80.4 & 88.3 & 91.9 & 93.7 & 95.3 & 100.0 & 100.4 & 101.4 & 104.6 & 105.3 & 108.4 & 111.1 & 111.6 & 110.9 & 111.1 & 111.5 & 113.9 & 115.0 & 117.7 & 123.1 \\
\hline Furniture and household operations & 18.1 & 90.5 & 92.5 & 94.9 & 96.9 & 98.5 & 100.0 & 102.4 & 104.3 & 107.1 & 108.1 & 109.1 & 113.6 & 118.0 & 120.9 & 124.0 & 126.2 & 128.6 & 128.8 & 131.3 & 134.5 \\
\hline Transpert and corrmunications & 8.4 & 95.7 & 96.0 & 97.4 & 99.4 & 101.1 & 100.0 & 100.9 & 101.6 & 103.0 & 103.3 & 103.9 & 106.2 & 108.3 & 109.6 & 111.8 & 114.4 & 117.3 & 125.3 & 128.4 & 132.5 \\
\hline Other goods and services & 10.5 & 92.2 & 93.2 & 94.6 & 96.5 & 98.1 & 100.0 & 101.3 & 101.8 & 103.9 & 104.2 & 105.5 & 106.3 & 109.4 & 110.7 & 112.5 & 113.3 & 114.5 & 115.7 & 116.6 & 116.6 \\
\hline \multicolumn{22}{|l|}{$\begin{array}{l}\text { Menorandun ittem: } \\
\text { Armual CPI inflation rates (in percent, ond of period) }\end{array}$} \\
\hline Armual CPI inflation rates (in percent, end of period) & & 9.3 & 9.9 & 8.5 & 9.2 & 8.6 & 8.8 & 8.9 & 7.8 & 7.9 & 7.3 & 6.8 & 9.1 & 9.7 & 8.9 & 9.3 & 6.8 & 6.3 & 6.3 & 5.5 & 6.0 \\
\hline
\end{tabular}

1) Covers all households in six lowland towns, itrchuding Masenu.

2 Since January 1994, rent has beon excluded from CPI calculations because of data collection probiens 
Table 5. Lesotho: Basic Monthly Minimum Wages, 1995-99 1/

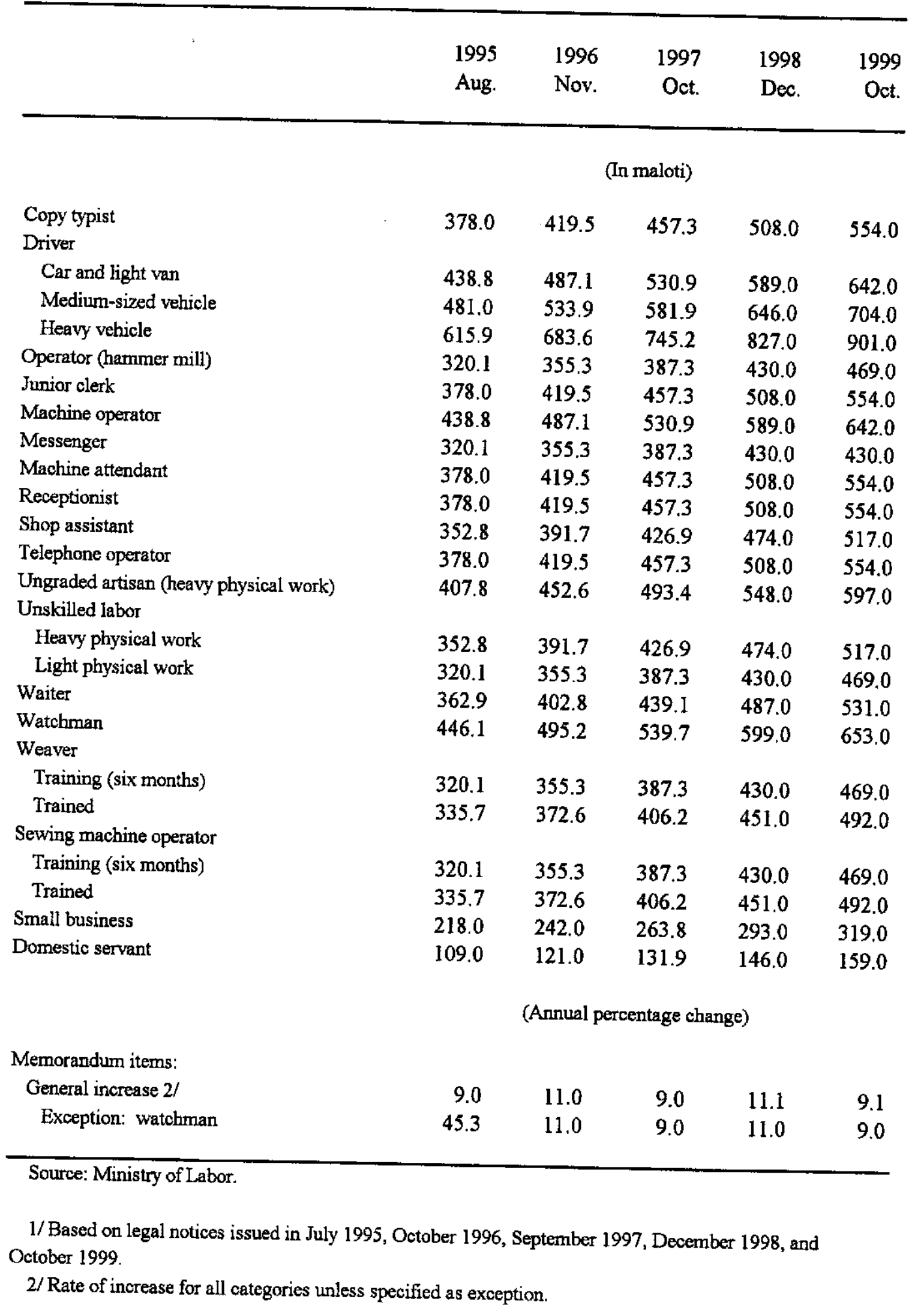


Table 6. Lesotho: Public Service Employment, 1995/96-1999/2000 1/

\begin{tabular}{|c|c|c|c|c|c|}
\hline : & $1995 / 96$ & $1996 / 97$ & $1997 / 98$ & $1998 / 99$ & $1999 / 00$ \\
\hline \multicolumn{6}{|l|}{ Established civil service $2 /$} \\
\hline Professional and administrative & 1,863 & 1,649 & 1,748 & 2,004 & 2,030 \\
\hline Executive & 5,073 & 3,407 & 3,453 & 3,639 & 3,667 \\
\hline Clerical & 11,054 & 13,442 & 13,540 & 14,822 & 14,993 \\
\hline Total & 17,990 & 18,498 & 18,741 & 20,465 & 20,690 \\
\hline \multicolumn{6}{|l|}{ Actual employment } \\
\hline Civil service $2 /$ & 16,780 & 17,685 & 18,436 & 18,436 & 18,271 \\
\hline Defense and public order & 4,881 & 4,942 & 4,942 & 6,394 & 6,288 \\
\hline Teachers & 9,601 & 9,868 & 10,116 & 10,209 & 10,425 \\
\hline Total & 31,262 & 32,495 & 33,494 & 35,039 & 34,984 \\
\hline
\end{tabular}

Source: Ministry of Public Service.

1/ Fiscal year is April-March.

2/ The established civil service posts exclude teachers, members of armed forces, and daily paid workers, but include chiefs, parliamentarians, senators, and statutory positions. 
Table 7. Lesotho: Central Government Operations, 1995/96-1999/2000 1/

\begin{tabular}{|c|c|c|c|c|c|}
\hline & 1995/96 & $1996 / 97$ & $1997 / 98$ & $1998 / 99$ & $1999 / 00$ \\
\hline : & \multicolumn{5}{|c|}{ (In millions of maloti) } \\
\hline Revenue & $1,685.3$ & $2,034.6$ & $2,247.0$ & $2,173.6$ & $2,312.8$ \\
\hline Tax revenue & $1,413.5$ & $1,553.8$ & $1,794.9$ & $1,694.7$ & $1,888.8$ \\
\hline Custorns revenue & 906.5 & $1,006.0$ & $1,172.7$ & $1,034.4$ & $1,183.1$ \\
\hline Noneustoms tax revenue & 507.0 & 547.8 & 622.2 & 661.3 & 705.7 \\
\hline Income taxes & 275.8 & 299.0 & $\mathbf{3 4 0 . 1}$ & 387.0 & 419.5 \\
\hline Sales tax & 174,0 & 193.9 & 223.5 & 233.3 & 238.0 \\
\hline Oit levy & 45.6 & 46.0 & 47.4 & 35.9 & 43.6 \\
\hline Other tax revenues & 11.6 & 8.9 & 11.2 & 5.1 & 4.6 \\
\hline Nontax revenue & 271.8 & 480.8 & 452.1 & 478.9 & 424.0 \\
\hline Water royaltics & 0.0 & 142.8 & 83.3 & 120.0 & 138.3 \\
\hline Interest received & 109.8 & 150.7 & 177.7 & 222.9 & 76.0 \\
\hline Other nontax revenues & 162.0 & 187.3 & 191.1 & 136.0 & 209.7 \\
\hline Total expenditure and net lending & $1,675.9$ & $2,052.7$ & $2,342.1$ & $2,438.4$ & $3,373.4$ \\
\hline Current expenditure & $1,118.1$ & $1,179.0$ & $1,473.8$ & $1,942.7$ & $2,318.5$ \\
\hline Wages and salaries & 518.7 & 604.4 & 721.3 & 837,6 & 835.9 \\
\hline Interest payments & 59.2 & 66.3 & 90.0 & 128.5 & 183.4 \\
\hline Of which: external interest & 32.0 & 36.6 & 59.9 & 96.2 & 101.6 \\
\hline Goods, services, and transfers & 540.2 & 508.3 & 662.5 & 976.6 & $1,299.2$ \\
\hline Goods and services & 394.0 & 268.2 & 323.6 & 653.8 & $1,028.8$ \\
\hline Transfers and subsidies & 146.2 & 240.1 & 338.9 & 322.8 & 270.4 \\
\hline Capital expenditure and net lending & 557.8 & 873.7 & 868.3 & 495.7 & 479.9 \\
\hline Domestically funded & 119.0 & 252.3 & 247.4 & 233.2 & 250.0 \\
\hline Of which : Development Fund & 71.2 & 86.5 & 33.0 & 55.8 & 51.0 \\
\hline Extemally funded & 438.8 & 621.4 & 620.9 & 262.5 & 229.9 \\
\hline Grant funded & 163.2 & 193.4 & 178.7 & 120.0 & 130.0 \\
\hline Loan funded & 275.6 & 428.0 & 442.2 & 142.5 & 99.9 \\
\hline Overall balance before grants & 9.4 & -18.1 & -95.1 & -264.8 & $-1,060.6$ \\
\hline Grants from abroad & 163.2 & 203.4 & 178.7 & 120.0 & 130.0 \\
\hline Overall balance after grants & 172.6 & 185.3 & 83.6 & -144.8 & -930.6 \\
\hline Total financing & -172.6 & -185.3 & -83.6 & 144.8 & 930.6 \\
\hline Financing abroad & 203.9 & 347.2 & 345.5 & 18.1 & -70.9 \\
\hline Loan drawings & 275.6 & 428.0 & 442.2 & 142.5 & 99.9 \\
\hline Amortization & -71.7 & -80.8 & -96.7 & -124.4 & $-170,8$ \\
\hline Domestic financing & -327.2 & -543.0 & -489.3 & 130.7 & $1,001.3$ \\
\hline Bank & -317.4 & -527.7 & -507.7 & 116.2 & 973.1 \\
\hline Nonbank & -9.8 & -15.3 & 18.4 & 14.5 & 28.2 \\
\hline \multirow[t]{2}{*}{ Residual } & -49.3 & 10.5 & 60.2 & -4.0 & 0.2 \\
\hline & \multicolumn{5}{|c|}{ (In percent of GDP, unless otherwise indicated) } \\
\hline Revenue & 47.5 & 48.3 & 47.2 & 42.8 & 40.4 \\
\hline Customs revenue & 25.5 & 23.9 & 24.6 & 20.4 & 20.6 \\
\hline Noncustoms tax revenuc & 14.3 & 13.0 & 13.1 & 13.0 & 12.3 \\
\hline Nontax revenue & 7.7 & 11.4 & 9.5 & 9,4 & 7.4 \\
\hline Total expenditure and net lending & 47.2 & 48.7 & 49.2 & 48.0 & 58.9 \\
\hline Current expenditure & 31.5 & 28.0 & 30.9 & 38.3 & 40.5 \\
\hline Wagea and saiaries & 14.6 & 14.3 & 15.1 & 16.5 & 14.6 \\
\hline Other expenditure & 16.9 & 13.6 & 15.8 & 21.8 & 25.9 \\
\hline Capital expenditure and net lending & 15.7 & 20.7 & 18.2 & 9.8 & 8.4 \\
\hline Overall balance before grants & 0.3 & -0.4 & -2.0 & -5.2 & -18.5 \\
\hline Grants from abroad & 4.6 & 4.8 & 3.8 & 2.4 & 2.3 \\
\hline Overall balance after grants & 4.9 & 4.4 & 1.8 & -2.9 & -16.2 \\
\hline Financing abroad & 5.7 & 8.2 & 7.3 & 0.4 & -1.2 \\
\hline Domestic bank financing & -8.9 & -12.5 & -10.7 & 2.3 & 17.0 \\
\hline \multicolumn{6}{|l|}{ Memorandum item: } \\
\hline GDP at market prices (in millions of maloci) & $3,549.9$ & $4,216.3$ & $4,764.2$ & $5,078.8$ & $5,730.9$ \\
\hline
\end{tabular}

Sources: Ministry of Finance; and staff ostimates.

1/ Fiscal year is April-March. 
Table 8. Lesotho: Government Revenue and Grants, 1995/96-1999/2000 1/

(In millions of maloti)

\begin{tabular}{|c|c|c|c|c|c|}
\hline & $1995 / 96$ & $1996 / 97$ & $1997 / 98$ & $1998 / 99$ & $1999 / 00$ \\
\hline Taxes on net income and profits & 275.8 & 299.0 & 340.1 & 387.0 & 419.5 \\
\hline Company tax & 56.1 & 52.6 & 69.7 & 65.0 & 58.5 \\
\hline Income tax (pay as you eam) & 195.1 & 204.6 & 221.7 & 278.0 & 306.5 \\
\hline Gaming tax & 2.5 & 2.9 & 3.0 & 1.7 & 3.8 \\
\hline Withhoiding tax & 18.4 & 23.4 & 26.7 & 28.8 & 40.2 \\
\hline Other income and profit taxes & 3.7 & 15.5 & 19.0 & 13.5 & 10.5 \\
\hline Taxes on goods and services & 220.7 & 241.6 & 273.3 & 270.9 & 283.2 \\
\hline Sales tax & 174.0 & 193.9 & 223.5 & 233.3 & 238.0 \\
\hline Trade licenses & 1.1 & 1.7 & 2.4 & 1.7 & 1.6 \\
\hline Petrol levy & 45.6 & 46.0 & 47.4 & 35.9 & 43.6 \\
\hline \multicolumn{6}{|l|}{ Taxes on international trade } \\
\hline and transactions & 906.5 & $1,006.0$ & $1,172.7$ & $1,034.4$ & $1,183.1$ \\
\hline Customs duties & 906.5 & $1,006.0$ & $1,172.7$ & $1,033.4$ & $1,183.1$ \\
\hline Livestock import levy & 0.0 & 0.0 & 0.0 & 1.0 & 0.0 \\
\hline Export duties on diamonds & 0.0 & 0.0 & 0.0 & 0.0 & 0.0 \\
\hline Other taxes & 10.5 & 7.2 & 8.8 & 2.4 & 3.0 \\
\hline Stamp duty & 1.4 & 2.0 & 2.1 & 0.6 & 0.9 \\
\hline Other taxes & 9.1 & 5.2 & 6.7 & 1.8 & 2.1 \\
\hline Total tax revenue & $1,413.5$ & $1,553.8$ & $1,794.9$ & $1,694.7$ & $1,888.8$ \\
\hline \multicolumn{6}{|l|}{$\begin{array}{l}\text { Administrative fees, charges, } \\
\text { and nonindustrial sales }\end{array}$} \\
\hline and nonindustrial sales & 85.0 & 82.0 & 106.9 & 67.8 & 142.7 \\
\hline Attestation fees & 1.0 & 0.5 & 0.8 & 0.6 & 0.6 \\
\hline Fines and forfeits & 1.1 & 3.2 & 4.3 & 3.9 & 6.4 \\
\hline Property and other income & 184.7 & 395.1 & 340.1 & 403.6 & 274.3 \\
\hline Interest on deposits & 109.8 & 150.7 & 177.7 & 222.9 & 76.0 \\
\hline Water royalties & 0.0 & 142.8 & 83.3 & 120.0 & 138.3 \\
\hline Rand monetary compensation & 28.6 & 64.7 & 34.6 & 43.0 & 42.0 \\
\hline Dividends & 17.5 & 15.6 & 14.6 & 9.1 & 2.4 \\
\hline Other property income & 28.8 & 21.3 & 29.9 & 8.6 & 15.6 \\
\hline Total nontax revenue & 271.8 & 480.8 & 452.1 & 478.9 & 424.0 \\
\hline Total revenue & $1,685.3$ & $2,034.6$ & $2,247.0$ & $2,173.6$ & $2,312.8$ \\
\hline Grants & 163.2 & 203.4 & 178.7 & 120.0 & 130.0 \\
\hline Total revenue and grants & $1,848.5$ & $2,238.0$ & $2,425.7$ & $2,293.6$ & $2,442.8$ \\
\hline
\end{tabular}

Sources: Ministry of Finance; and staff estimates.

1/ Fiscal year is April-March. 
Table 9. Lesotho: Southern African Customs Union (SACU) Operations, 1995/96-1999/2000

\begin{tabular}{llllll}
\hline Revenue Year 1/ & $1995 / 96$ & $1996 / 97$ & $1997 / 98$ & $1998 / 99$ & $1999 / 00$ \\
Data Year 2/ & $1993 / 94$ & $1994 / 95$ & $1995 / 96$ & $1996 / 97$ & $1997 / 98$ \\
\hline
\end{tabular}

(In percent)

Basic rate 3/

Revenue ("compensation") rate 4/

Stabilization factor 5 /

Stabilized rate (calculated) $6 /$

Stabilized rate (actual) $7 /$

\begin{tabular}{|c|c|c|c|c|}
\hline 9.1 & 8.0 & 7.5 & 6.5 & 6.2 \\
\hline 12.9 & 11.4 & 10.7 & 9.2 & 8.7 \\
\hline 3.5 & 4.3 & 4.7 & 5.4 & 5.7 \\
\hline 16.5 & 15.7 & 15.3 & 14.6 & 14.4 \\
\hline 17.0 & 17.0 & 17.0 & 17.0 & 17 \\
\hline
\end{tabular}

Dutiable base 8/

Growth rate (in percent)

(In millions of maloti, unless otherwise specified)

First estimate (payment) $9 /$

First adjustment (payment) 10/

Final adjustment (payment) 11/

$\begin{array}{rrrrr}4,224.6 & 4,787.3 & 5,561.6 & 5,433.0 & 6,260.5 \\ 15.5 & 13.3 & 16.2 & -2.3 & 15.2 \\ & & & & \\ 718.2 & 813.8 & 945.5 & 923.6 & 1,064.3 \\ 188.3 & 192.2 & 227.3 & 109.8 & 118.8 \\ 0.0 & 0.0 & 0.0 & 0.1 & 0.0\end{array}$

Actual receipts 12/

Growth rate (in percent)

$\begin{array}{rrrrr}906.5 & 1,006.0 & 1,172.7 & 1,033.4 & 1,183.1 \\ .7 .8 & 11.0 & 16.6 & -11.9 & 14.5\end{array}$

Memorandum item:

First estimate based on basic rate only 13/

$384.4 \quad 383.0 \quad 417.1 \quad 353.1$

388.2

Sources: Department of Customs and Excise; and staff estimates.

1/ Fiscal year (April-March) in which indicated revenue payments are received.

2/ Fiscal year of data on which calculations are based (rates and dutiable base).

3/ Customs and excise revenues as percent of dutiable base (imports and excisable production, and duties) for

Southern African Customs Union as a whole (data year).

4/ Basic rate multiplied by 1.42 , as initial compensation for disadvantages to smaller members.

$5 /$ One-half of difference between 20 percent and revenue (compensation) rate.

6/ Revenue (compensation) rate plus stabilization factor.

7/ At least 17.0 percent and no more than 23.0 percent; the calculated stabilized rate applies if it falls between 17 percent and 23 percent. In recent years, the lower limit of 17.0 percent has been the operative rate applied to the
dutiable base.

8/ Lesotho's imports (c.i.f. and duty paid, adjusted to include electricity, estimated border shopping, etc.), excisable goods produced and consumed, and duties collected in the data year.

9/ Stabilized rate (actual) times dutiable base. Referred to as "accrued receipts" of data year.

10/ Stabilized rate (actual) times increase in dutiable base from two years earlier (as allowance for growth in dutiable base to revenue year).

11/ Minor adjustments made to account for revisions in base data, usually of previous data year. Calculated here as a residual.

12/ As reported in government revenue data.

13/ Basic rate times dutiable base. Referred to as "accrued receipts based on basic rate only." 
Table 10. Lesotho: Economic Classification of Government Expenditure, 1995/96-1999/2000 1/ (In millions of maloti)

\begin{tabular}{|c|c|c|c|c|c|}
\hline & $1995 / 96$ & $1996 / 97$ & $1997 / 98$ & $1998 / 99$ & $1999 / 00$ \\
\hline Current expenditure & $1,118.1$ & $1,179.0$ & $1,473.8$ & $1,942.7$ & $2,318.5$ \\
\hline Goods and services & 912.7 & 872.6 & $1,044.9$ & $1,341.7$ & $1,864.7$ \\
\hline Wages and salaries & 518.7 & 604.4 & 721.3 & 837.6 & 835.9 \\
\hline Other purchases & 394.0 & 268.2 & 323.6 & 504.1 & $1,028.8$ \\
\hline Interest payments & 59.2 & 66.3 & 90.0 & 128.5 & 183.4 \\
\hline External & 32.0 & 36.6 & 59.9 & 96.2 & 101.6 \\
\hline Domestic & 27.2 & 29.7 & 30.1 & 32.3 & 81.8 \\
\hline Subsidies and transfers & 146.2 & 240.1 & 338.9 & 472.6 & 270.4 \\
\hline Pensions & 20.6 & 29.8 & 22.9 & 53.7 & 53.7 \\
\hline Subventions and transfers & 125.6 & 210.3 & 316.0 & 418.9 & 418.9 \\
\hline Of which: social safety net & 8.0 & 6.0 & 5.0 & 6.0 & 6.0 \\
\hline Capital expenditure and net lending & 557.8 & 873.7 & 868.3 & 495.7 & $1,054.9$ \\
\hline Acquisition of assets & 512.5 & 846.6 & 830.3 & 360.7 & 360.7 \\
\hline Transfers and subventions & 45.3 & 27.1 & 38.0 & 135.0 & 135.0 \\
\hline Net lending & $\cdots$ & $\cdots$ & $\ldots$ & $\cdots$ & $\ldots$ \\
\hline Total expenditure and net lending & $1,675.9$ & $2,052.7$ & $2,342.1$ & $2,438.4$ & $3,373.4$ \\
\hline
\end{tabular}

Sources: Ministry of Finance; and staff estimates.

1/ Fiscal year is April-March. 
Table 11. Lesotho: Functional Classification of Govemment Expenditure, 1995/96-1999/2000 I/

(In millions of maloti)

\begin{tabular}{|c|c|c|c|c|c|}
\hline$\therefore$ & $1995 / 96$ & $1996 / 97$ & $1997 / 98$ & $1998 / 99$ & $1999 / 00$ \\
\hline Current expenditure & $1,118,1$ & $1,179.0$ & $1,473.8$ & $1,942.7$ & $2,318.5$ \\
\hline General public service & 336.3 & 369.1 & 550.0 & 801.4 & 957.3 \\
\hline Public order, safety, and defense & 187.3 & 203.0 & 303.7 & 390.9 & 469.1 \\
\hline Of which: defense & 96.9 & 109.8 & 143.4 & 159.8 & 160.6 \\
\hline Other services & 149.0 & 166.1 & 246.3 & 410.5 & 488.2 \\
\hline Health, social security, and welfare & 128.7 & 131.3 . & 151.2 & 210.3 & 200.4 \\
\hline Education and community sorvices & 360.5 & 370.9 & 453.6 & 532.2 & 585.8 \\
\hline Economic services & 197.4 & 214.7 & 260.0 & 219.0 & $28 \mathrm{I} .3$ \\
\hline Agriculture and nural development & 105.2 & 107.8 & 129.2 & 80.2 & 99.0 \\
\hline Commerce, tourism, and industry & 17.4 & 19.8 & 21.2 & 29.0 & 34.1 \\
\hline Water, energy, and mining & 12.3 & 15.5 & 20.4 & 24.0 & 40.4 \\
\hline Roads & 53.7 & 55.8 & 74.5 & 69.7 & 66.2 \\
\hline Other transport and communication & 8.8 & 15.8 & 14.7 & 16.1 & 41.6 \\
\hline Unallocable and other purpones $2 /$ & 95.2 & 93.0 & 59.0 & 179.8 & 293.7 \\
\hline Capital expenditure and net lending & $\$ 57.8$ & 873.7 & 868.3 & 495.8 & $1,054.9$ \\
\hline General public service & 42.2 & 119.1 & 123.4 & 34.7 & 666.6 \\
\hline Public order, safety, and deferse & 4.1 & 17.2 & 23.2 & 3.7 & 20.7 \\
\hline Of which: defense & 0.5 & 0.0 & 0.0 & 0.0 & 13.9 \\
\hline Other services & 38.1 & 101.9 & 100.2 & 31.0 & 645.9 \\
\hline Health, social security, and welfare & 46.5 & 73.1 & 39.2 & 17.2 & 43.5 \\
\hline Education and community services & 77.0 & 101.7 & 70.6 & 120.4 & 108.5 \\
\hline Economic services & 448.9 & 577.2 & 635.1 & 323.5 & 236.3 \\
\hline Agriculture and rural development & 102.7 & 115.6 & 104.3 & 45.2 & 52.7 \\
\hline Commerce, tourism, and industry & 27.4 & 0.9 & 8.1 & 20.4 & 17.2 \\
\hline Water, onergy, and mining & 215.1 & 266.2 & 202.5 & 97.7 & 99.2 \\
\hline Roads & 99.8 & 116.7 & 291.2 & 158.6 & 50.7 \\
\hline Other transport and communication & 3.9 & 77.8 & 29.0 & 1.6 & 16.3 \\
\hline Unallocable and other purposes $2 /$ & -56.8 & 2.6 & 0.0 & 0.0 & 0.0 \\
\hline Total expenditure and net lending & $1,675.9$ & $2,052.7$ & $2, \mathbf{3 4 2 . 1}$ & $2,438,5$ & $3,373.4$ \\
\hline General public service & 378.5 & 488.2 & 673.4 & 836.1 & $1,623.9$ \\
\hline Public order, axfety, and defense & 191.4 & 220.2 & 326.9 & 394.6 & 489.8 \\
\hline Of which : defense & 97.4 & 109.8 & 143.4 & 159.8 & 174.5 \\
\hline Other services & 187.1 & 268.0 & 346.5 & 441.5 & $1,134.1$ \\
\hline Health, social security, and welfare & 175.2 & 204.4 & 190.4 & 227.5 & 243.9 \\
\hline Education and community services & 437.5 & 472.6 & 524.2 & 652.6 & 694.3 \\
\hline Economic services & 646.3 & 791.9 & 895.1 & 542.5 & 517.6 \\
\hline Agriculture and rural development & 207.9 & 223.4 & 233.5 & 125.4 & 151.7 \\
\hline Commerce, tourism, and industry & 44.8 & 20.7 & 29.3 & 49.4 & 51.3 \\
\hline Water, cnergy, and mining & 227.4 & 281.7 & 222.9 & 121.7 & 139.6 \\
\hline Roadr & 153.5 & 172.5 & 365.7 & 228.3 & 116.9 \\
\hline Other transport and communication & 12.7 & 93.6 & 43.7 & 17.7 & 58.1 \\
\hline Unallocable and other purposes $2 /$ & 38.4 & 95.6 & 59.0 & 179.8 & 293.7 \\
\hline
\end{tabular}


Table 12. Lesotho: Outstanding Government Domestic Debt by Instrument and Holder, 1995-99

(In millions of maloti)

\begin{tabular}{|c|c|c|c|c|c|}
\hline & 1995 & 1996 & 1997 & 1998 & 1999 \\
\hline & \multicolumn{5}{|c|}{ March 31} \\
\hline \multicolumn{6}{|l|}{ Commercial banks } \\
\hline Gross lending $\mathrm{l} /$ & 93.5 & 65.8 & 63.5 & 58.8 & 36.0 \\
\hline Long term & 5.7 & 6.2 & 4.1 & 3.6 & 3.9 \\
\hline Bonds & 0.0 & 0.0 & 0.0 & 0.0 & 0.0 \\
\hline Loans & 5.7 & 6.2 & 4.1 & 3.6 & 3.9 \\
\hline Short term & 87.8 & 59.6 & 59.4 & 55.2 & 32.1 \\
\hline Loans & 0.5 & 0.1 & 0.0 & 0.0 & 0.0 \\
\hline Treasury bills & 87.3 & 59.5 & 59.4 & 55.2 & 32.1 \\
\hline Government deposits $(-)$ & -41.7 & -37.5 & -50.9 & -48.6 & -66.4 \\
\hline Net total & 51.8 & 28.3 & 12.6 & 10.2 & -30.4 \\
\hline \multicolumn{6}{|l|}{ Central bank } \\
\hline Gross lending 1/ & 144.9 & 233.3 & 152.0 & 61.4 & 71.1 \\
\hline Long term & 0.9 & 0.5 & 0.0 & 0.0 & 0.0 \\
\hline Bonds & 0.0 & 0.0 & 0.0 & 0.0 & 0.0 \\
\hline Loans & 0.9 & 0.5 & 0.0 & 0.0 & 0.0 \\
\hline Short term & 144.0 & 232.8 & 152.0 & 61.4 & 71.1 \\
\hline Loans & 127.1 & 184.2 & 124.8 & 63.4 & 64.7 \\
\hline Treasury bills & 16.9 & 48.6 & 27.2 & -2.0 & 6,4 \\
\hline Government deposits (-) & $-1,068.4$ & $-1,441.7$ & $-1,873.5$ & $-2,231.6$ & $-2,086.2$ \\
\hline Net total & -923.5 & $-1,208.4$ & $-1,721.5$ & $-2,170.2$ & $-2,015.1$ \\
\hline \multicolumn{6}{|l|}{ Nonbank 2/ } \\
\hline Long term & 0.0 & 0.0 & 0.0 & 0.0 & 0.0 \\
\hline Bonds & 0.0 & 0.0 & 0.0 & 0.0 & 0.0 \\
\hline Other & 0.0 & 0.0 & 0.0 & 0.0 & 0.0 \\
\hline Short term & 57.8 & 43.3 & 67.5 & 100.9 & 115.5 \\
\hline Treasury bills & 49.9 & 46.0 & 67.5 & 100.9 & 115.5 \\
\hline Compulsory savings & 7.9 & -2.7 & 0.0 & 0.0 & 0.0 \\
\hline Promissory notes & 0.0 & 0.0 & 0.0 & 0.0 & 0.0 \\
\hline Total nonbank & 57.8 & 43.3 & 67.5 & 100.9 & 115.5 \\
\hline Total domestic debt, net & -813.9 & $-1,136.8$ & $-1,641.4$ & $-2,059.1$ & $-1,930.0$ \\
\hline Gross debt outstanding & 296.2 & 342.4 & 283.0 & 221.1 & 222.6 \\
\hline Government deposits $(-)$ & $-1,110.1$ & $-1,479.2$ & $-1,924.4$ & $-2,280.2$ & $-2,152.6$ \\
\hline
\end{tabular}

Source: Central Bank of Lesotho.

1/ Data differ slightly in coverage from banking statistics and may not fully reflect revisions made there. 2/ The nonbank sector includes insurance, bank pension schemes, public servants' promissory notes and compulsory savings, and public enterprises, as well as the general public. 
Table 13. Losotho: Monetary Survey, March 1995-September 2000

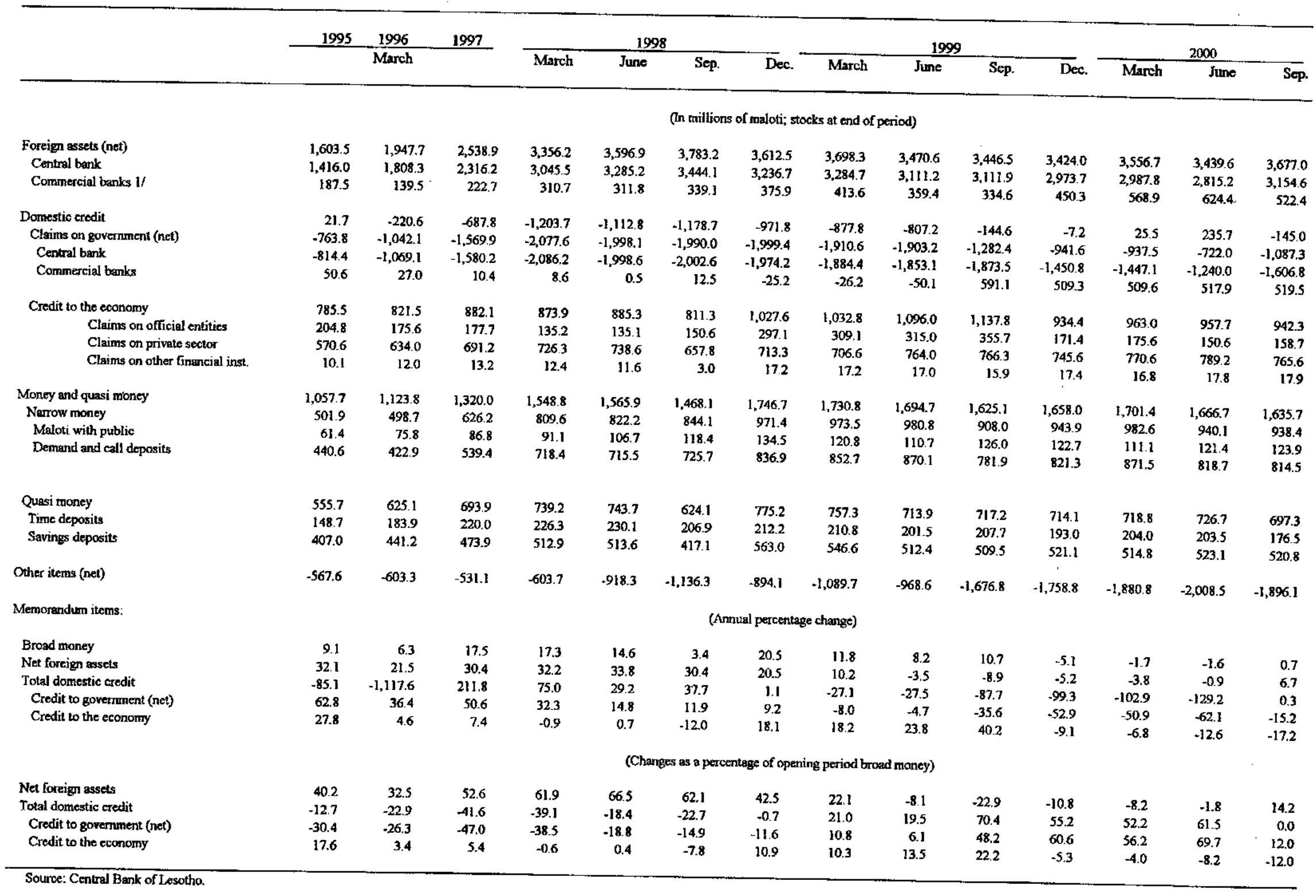

IIncludes rand notes and coins 
Table 14. Lesotho: Assets and Liabilities of the Central Bank of Lesotho, March 1995-September 2000

(In millions of maloti; end of period)

\begin{tabular}{|c|c|c|c|c|c|c|c|c|c|c|c|c|c|c|}
\hline & 1995 & 1996 & 1997 & \multicolumn{4}{|c|}{1998} & \multicolumn{4}{|c|}{1999} & \multicolumn{3}{|c|}{2000} \\
\hline & & March & & March & June & Sep. & Dec. & March & June & Sep. & Dec. & March & June & Sep. \\
\hline Foreign assets & $1,421.7$ & $1,815.0$ & $2,322.4$ & $3,052.4$ & $3,291.8$ & $3,450.8$ & $3,381.6$ & $3,422.3$ & $3,235.4$ & $3,226.9$ & $3,085.5$ & $3,090.9$ & $2,905.3$ & $3,245.6$ \\
\hline Claims on govermment & 297.8 & 372.5 & 293.3 & 145.4 & 270.6 & 260.2 & 145.9 & 201.8 & 161.5 & 144.3 & 110.4 & 247.7 & 130.0 & 109.2 \\
\hline Unclassified assets & -95.6 & -87.3 & -47.7 & 65.0 & 16.8 & -50.5 & 58.6 & 45.1 & 69.1 & 78.4 & 74.6 & 61.9 & 51.1 & 138.7 \\
\hline Total assets $=$ total liabilities & $1,623.9$ & $2,100.2$ & $2,568.0$ & $3,262.8$ & $3,579.2$ & $3,660.5$ & $3,586.1$ & $3,669.2$ & $3,466.0$ & $3,449.6$ & $3,270.5$ & $3,400.5$ & $3,086.3$ & $3,493.6$ \\
\hline Reserve money & 198.9 & 236.5 & 247.8 & 406.1 & 429.6 & 523.5 & 526.9 & 514.6 & 454.5 & 479.1 & 702.3 & 592.4 & 536.0 & 556.4 \\
\hline Maloti in circulation & 80.4 & 97,8 & 113.9 & 118.0 & 135.1 & 142.7 & 165.0 & 147.3 & 136.4 & 151.4 & 158.0 & 135.7 & 142.7 & 145.7 \\
\hline Bankers' deposits & 118.5 & 138.7 & 133.9 & 288.0 & 294,5 & 380.8 & 361.9 & 367.3 & 318.2 & 327.7 & 544.3 & 456.7 & 393.3 & 410.7 \\
\hline Foreign monetary liabilities & 5.7 & 6.7 & 6.2 & 6.9 & 6.6 & 6.6 & 145.0 & 137.6 & 124.2 & 115.0 & 111.8 & 103.1 & 90.0 & 91.1 \\
\hline Govemment deposits & $1,112.1$ & $1,441.7$ & $1,873.5$ & $2,231.6$ & $2,269.3$ & $2,262.8$ & $2,120.1$ & $2,086.2$ & $2,014.7$ & $2,017.8$ & $1,561.2$ & $1,694.9$ & $1,369.9$ & $1,716.0$ \\
\hline Capital accounts & 73.2 & 71.4 & 79.7 & 83.1 & 86.8 & 93.3 & 94.9 & 81.8 & 82.7 & 96.0 & 96.4 & 98.1 & 95.1 & 99.8 \\
\hline Capital and reserves & 54.1 & 50.1 & 56.1 & 60.3 & 64.0 & 63.3 & 64.0 & 64.0 & 64.9 & 64.9 & 64.9 & 64.9 & 64.9 & 64.9 \\
\hline Allocation of SDRs & 19.1 & 21.3 & 23.5 & 22.8 & 22.8 & 29.9 & 30.9 & 17.8 & 17.8 & 31.2 & 31.6 & 33.2 & 30.3 & 35.0 \\
\hline Unclassified liabilities & 233.9 & 343.9 & 360.8 & 535.2 & 786.9 & 774.3 & 699.2 & 849.0 & 790.0 & 741.7 & 798.7 & 912.1 & 995.2 & $1,030.2$ \\
\hline
\end{tabular}


Table 1S. Lesotho: Assets and Liabilities of Commercial Banks, March 1995-Septenber 2000

(In millions of maloti; end of period)

\begin{tabular}{|c|c|c|c|c|c|c|c|c|c|c|c|c|c|c|}
\hline & 1995 & 1996 & 1997 & \multicolumn{4}{|c|}{1998} & \multicolumn{4}{|c|}{1999} & \multicolumn{3}{|c|}{2000} \\
\hline & & March & & March & June & Sep. & Dec. & March & June & Sep. & Dec. & March & June & Sep. \\
\hline Reserves & 92.8 & 151.3 & 146.6 & 313.3 & 317.0 & 326.5 & 489.2 & 384.2 & 347.6 & 346.0 & $\$ 73.7$ & 491.5 & 415.1 & 423.2 \\
\hline Maloti on hand & 12.5 & 15.7 & 20.4 & 18.3 & 19.4 & 17.0 & 18.9 & 21.2 & 18.5 & 18.9 & 26.1 & 18.7 & 16.3 & 16.6 \\
\hline Rand on hand & 6.6 & 6.3 & 6.7 & 8.6 & 9.0 & 7.3 & 11.6 & 5.3 & 7.2 & 6.4 & 9.3 & 5.9 & 5.1 & 5.2 \\
\hline Balances with central bank & 73.7 & 129.4 & 119.5 & 286.4 & 288.5 & 302.2 & 458.7 & 357.7 & 322.0 & 320.6 & 538.3 & 466.9 & 393.8 & 401.4 \\
\hline Foreign assets & 217.4 & 200.7 & 256.0 & 338.7 & 356.9 & 368.0 & 423.1 & 461.5 & 406.8 & 386.9 & 490.2 & 650.6 & 672.8 & 566.4 \\
\hline Claims on government & 92.3 & 64.4 & 61.2 & 57.2 & 48.6 & 59.0 & 51.5 & 35.2 & 21.6 & 655.7 & 586.4 & 584.1 & 587.4 & 591.0 \\
\hline Claims on statutory bodies & 204.8 & 175.6 & 177.7 & 135.2 & 135.1 & 150.6 & 297.1 & 309.1 & 315.0 & 355.7 & 171.4 & 175.6 & 150.6 & 158.7 \\
\hline Claims on private sector & 580.7 & 646.0 & 704.4 & 738.7 & 750.2 & 660.7 & 730.5 & 723.7 & 781.0 & 782.2 & 763.0 & 787.4 & 807.0 & 783.5 \\
\hline Unclassified assets & 247.4 & 417.2 & 334.2 & 266.3 & 253.7 & 201.8 & 173.9 & 292.1 & 392.0 & 389.7 & 427.8 & 920.2 & 979.3 & 251.0 \\
\hline Total assets $=$ total liabilities & $1,435.2$ & $1,655.2$ & $1,680.2$ & $1,849.4$ & $1,861.6$ & $1,766.6$ & $2,165.3$ & $2,205.8$ & $2,264.0$ & $2,916.0$ & $3,012.5$ & $3,609.4$ & $3,612.2$ & $2,773.9$ \\
\hline Demand and call deposits $1 /$ & 440.6 & 422.9 & 539.4 & 718.4 & 715.5 & 725.7 & 836.9 & 852.7 & 870.1 & 781.9 & 821.3 & 871.5 & 818.7 & 814.5 \\
\hline Savings and time deposits $1 /$ & 555.7 & 625.1 & 693.9 & 739.2 & 743.7 & 624.1 & 775.2 & 757.3 & 713.9 & 717.2 & 714.1 & 718.8 & 726.7 & 697.3 \\
\hline Savings deposits & 407.0 & 441.2 & 473.9 & $\$ 12.9$ & 513.6 & 417.1 & 563.0 & 546.6 & 512.4 & 509.5 & 521.1 & 514.8 & 523.1 & 520.8 \\
\hline Time deposits & 148.7 & 183.9 & 220.0 & 226.3 & 230.1 & 206.9 & 212.2 & 210.8 & 201.5 & 207.7 & 193.0 & 204.0 & 203.5 & 176.5 \\
\hline Of which: Miners' Deferred Pay Fund & 48.7 & 43.5 & 51.2 & 47.9 & 47.9 & 47.9 & 40.2 & 37.2 & 32.3 & 36.2 & 35.0 & 48.1 & 48.9 & 42.5 \\
\hline Foreign liabilities & 29.8 & 61.2 & 33.4 & 28.0 & 45.1 & 28.9 & 47.2 & 47.9 & 47.4 & 52.3 & 39.9 & 81.7 & 48.4 & 44.0 \\
\hline Government deposits & 41.7 & 37.4 & 50.9 & 48.6 & 48.1 & 46.5 & 76.8 & 61.4 & 71.6 & 64.5 & 77.1 & 74.4 & 69.4 & 71.5 \\
\hline Capital accounts & 238.8 & 212.2 & 228.8 & 150.3 & 166.4 & 243.9 & 145.4 & 143.7 & 84.4 & 724.2 & 760.7 & 818.6 & 829.5 & 864.7 \\
\hline Unclassified liabilities & 128.7 & 296.4 & 134.0 & 164.8 & 142.7 & 97.7 & 283.8 & 343.0 & 476.5 & 575.9 & 599.4 & $1,044.3$ & $1,119.5$ & 281.8 \\
\hline
\end{tabular}

1/ Excludes Miners' Deferred Pay Fund and nonresidents' deposits. 
Table 16. Lesotho: Principal Aggregates of Commercia]

Banks' Operations, March 1994-September 2000

(In millions of maloti, unless otherwise specified; end of period)

\begin{tabular}{|c|c|c|c|c|c|}
\hline & Deposits 1/ & $\begin{array}{r}\text { Loans and } \\
\text { Advances } 2 /\end{array}$ & $\begin{array}{l}\text { Credit- } \\
\text { Deposit } \\
\text { Ratio 3/ }\end{array}$ & $\begin{array}{r}\text { Liquid } \\
\text { Assets } 4 /\end{array}$ & $\begin{array}{r}\text { Liquidity } \\
\text { Ratio 5/ }\end{array}$ \\
\hline \multicolumn{6}{|l|}{$\begin{array}{c}19946 / \\
\text { March }\end{array}$} \\
\hline March & 870.0 & 602.7 & 69.3 & 209.3 & 24.1 \\
\hline June & 891.9 & 717.4 & 80.4 & 189.1 & 21.2 \\
\hline September & 897.8 & 765.3 & 85.2 & 166.9 & 18.6 \\
\hline December & 941.8 & 728.0 & 77.3 & 278.1 & 29.5 \\
\hline \multicolumn{6}{|l|}{$\begin{array}{l}1995 \\
\text { March }\end{array}$} \\
\hline March & 947.6 & 772.9 & 81.6 & 178.8 & 18.9 \\
\hline June & 888.7 & 806.4 & 90.7 & 195.2 & 22.0 \\
\hline September & 933.4 & 831.1 & 89.0 & 198.9 & 21.3 \\
\hline December & 999.6 & 817.6 & 81.8 & 233.3 & 23.3 \\
\hline \multicolumn{6}{|l|}{1996} \\
\hline March & $1,004.4$ & 807.7 & 80.4 & 209.5 & 20.9 \\
\hline June & $1,076.8$ & 748.8 & 69.5 & 247.5 & 23.0 \\
\hline September & $1,088.2$ & 786.8 & 72.3 & 198.8 & 18.3 \\
\hline December & $1,191.8$ & 808.2 & 67.8 & 316.5 & 26.6 \\
\hline \multicolumn{6}{|l|}{$\begin{array}{l}1997 \\
\text { March }\end{array}$} \\
\hline March & $1,182.0$ & 868.1 & 73.4 & 203.8 & 17.2 \\
\hline June & $1,233.0$ & 865.6 & 70.2 & 234.0 & 19.0 \\
\hline September & $1,282.1$ & 908.3 & 70.8 & 304.6 & 23.8 \\
\hline December & $1,311.3$ & 856.1 & 65.3 & 314.8 & 24.0 \\
\hline \multicolumn{6}{|l|}{1998} \\
\hline March & $1,409.7$ & 859.9 & 61.0 & 366.9 & 26.0 \\
\hline June & $1,411.3$ & 871.4 & 61.7 & 362.0 & 25.7 \\
\hline September & $1,301.8$ & 797.3 & 61.2 & 382.0 & 29.3 \\
\hline December & $1,572.0$ & $1,025.7$ & 65.2 & 537.2 & 34.2 \\
\hline \multicolumn{6}{|l|}{1999} \\
\hline March & $1,572.8$ & $1,031.1$ & 65.6 & 415.6 & 26.4 \\
\hline June & $1,551.7$ & $1,094.3$ & 70.5 & 368.9 & 23.8 \\
\hline September & $1,462.9$ & $1,101.1$ & 75.3 & 941.2 & 64.3 \\
\hline December & $1,500.4$ & 899.4 & 59.9 & $1,159.8$ & 77.3 \\
\hline \multicolumn{6}{|l|}{2000} \\
\hline March & $1,542.2$ & 928.0 & 60.2 & $1,075.3$ & 69.7 \\
\hline June & $1,496.5$ & 922.7 & 61.7 & $1,002.0$ & 67.0 \\
\hline September & $1,469.4$ & 907.3 & 61.7 & $1,013.7$ & 69.0 \\
\hline
\end{tabular}

1/ Excludes Miners' Deferred Pay Fund and deposits of nonresidents.

2/ Excludes loans and advances to nonresidents.

3/ Loans and advances as a percentage of deposits.

4/ Cash reserves, call or demand deposits with benks in the Common Monetary Area, and short-term government securities.

5/ Liquid assets as percentage of deposits.

6/ From 1994 onward, loans and advances include mortgages. 
Table 17. Lesotho: Sectoral Distribution of Commercial Bank Credit to the Private Scctor and Statutory Bodies, March 1995-September $2000 \mathrm{I} /$

(In millions of maloti; end of period)

\begin{tabular}{|c|c|c|c|c|c|c|c|c|c|c|c|c|c|c|}
\hline & \multirow[t]{2}{*}{1995} & \multirow{2}{*}{$\frac{1996}{\text { March }}$} & \multirow[t]{2}{*}{1997} & \multicolumn{4}{|c|}{1998} & \multicolumn{4}{|c|}{1999} & \multicolumn{3}{|c|}{2000} \\
\hline & & & & March & June & Sep. & Dec. & March & June & Sep. & Dec. & March & June & Sep. \\
\hline Agriculture & 36.4 & 39.7 & 41.0 & 52.5 & 50.1 & 42.8 & 14.1 & 15.0 & 4.4 & 1.0 & 1.0 & 1.0 & 1.0 & 0.9 \\
\hline Mining and quarrying & 0.7 & 0.9 & 0.8 & 5.1 & 1.7 & 0.9 & 0.9 & 0.8 & 0.8 & 0.8 & 0.9 & 0.9 & 0.9 & 0.8 \\
\hline Manufacturing & 63.6 & 62.8 & 67.6 & 73.6 & 77.9 & 80.4 & 63.8 & 82.7 & 54.3 & 53.3 & 56.2 & 54.2 & 57.9 & 58.3 \\
\hline Electricity, gas, and water & 9.2 & 70.3 & 76.4 & 98.6 & 81.7 & 90.5 & 132.6 & 88.4 & 32.5 & 123.8 & 69.6 & 46.6 & 48.9 & 42.7 \\
\hline Construction & 97.7 & 146.8 & 221.0 & 201.6 & 228.4 & 164.6 & 153.1 & 199.2 & 277.7 & 181.6 & 52.4 & 78.6 & 50.5 & 52.6 \\
\hline Trade, hotels, and restaurants & 76.9 & 64.4 & 68.5 & 65.4 & 76.2 & 72.8 & 48.2 & 69.1 & 49.2 & 62.7 & 57.5 & 65.0 & 73.6 & 87.9 \\
\hline Transport, storage, and communications & 57.1 & 50.6 & 57.5 & 76.7 & 71.3 & 63.4 & 55.0 & 58.5 & 36.0 & 98.4 & 83.0 & 87.3 & 89.7 & 101.0 \\
\hline $\begin{array}{l}\text { Nonbank financial institutions, real estate, } \\
\text { and business services }\end{array}$ & & & & & & & & & & & & & & \\
\hline $\begin{array}{l}\text { and business services } \\
\text { Community, social, and personal services }\end{array}$ & 40.1 & $\begin{array}{l}42.7 \\
36.1\end{array}$ & $\begin{array}{l}51.3 \\
37.5\end{array}$ & $\begin{array}{l}63.3 \\
66.0\end{array}$ & $\begin{array}{l}62.5 \\
75.9\end{array}$ & $\begin{array}{l}54.7 \\
61.4\end{array}$ & $\begin{array}{l}53.3 \\
57.9\end{array}$ & $\begin{array}{l}54.7 \\
57.7\end{array}$ & $\begin{array}{r}121.4 \\
58.7\end{array}$ & 31.9 & $\begin{array}{l}39.8 \\
64.6\end{array}$ & 39.7 & 32.4 & 20.8 \\
\hline $\begin{array}{l}\text { Community, social, and personal services } \\
\text { Personal loans }\end{array}$ & & $\begin{array}{r}36.1 \\
198.7\end{array}$ & $\begin{array}{r}37.5 \\
272.1\end{array}$ & 66.0 & $\begin{array}{r}75.9 \\
270.5\end{array}$ & 61.4 & 57.9 & 57.7 & 58.7 & 74.2 & 64.6 & 58.9 & 61.4 & 64.2 \\
\hline Personal loans & $\begin{array}{r}184.1 \\
0.0\end{array}$ & 198.7 & 222.1 & 222.8 & 228.5 & 231.6 & 358.1 & 366.2 & 392.2 & 390.8 & 390.8 & 412.9 & 406.8 & 395.3 \\
\hline Other $2 I$ & 0.0 & -0.1 & 0.1 & -89.1 & -105.5 & -74.8 & -0.1 & -50.0 & -21.5 & 0.7 & 0.1 & 0.0 & 15.7 & -1.2 \\
\hline Total claims on the economy & 596.5 & 713.1 & 843.8 & 836.4 & 848.6 & 788.3 & 936.9 & 942.4 & 1005.7 & 1019.2 & 815.9 & 845.1 & 838.8 & 823.3 \\
\hline Private sector & 558.0 & 620.2 & 677.2 & 712.3 & 724.7 & 643.8 & 711.4 & 704.9 & 762.3 & 729.6 & 710.6 & 735.6 & 754.2 & 730.6 \\
\hline Business enterprises & 374.0 & 421.5 & 455.1 & 489.5 & 496.2 & 412.2 & 353.3 & 338.6 & 370.1 & 338.8 & 319.8 & 322.6 & 347.5 & 335.3 \\
\hline Personal loans & 184.1 & 198.7 & 222.1 & 222.8 & 228.5 & 231.6 & 358.1 & 366.2 & 392.2 & 390.8 & 390.8 & 412.9 & 406.8 & 395.3 \\
\hline Statutory bodies & 38.5 & 92.8 & 166.6 & 124.0 & 124.0 & 144.5 & 225.5 & 237.5 & 243.4 & 289.6 & 105.3 & 109.5 & 84.6 & 92.7 \\
\hline
\end{tabular}

1/ Does not include investments and certain securities.

2/Calculated as residual. 
Table 18. Lesotho: Interest Rates Paid by the Central Bank on Commercial Bank Deposits, March 1994-September 2000

(In percent per annum; end of period)

\begin{tabular}{|c|c|c|c|c|c|}
\hline & Call & 31 Days & 88 Days & 6 Months & 1 Year \\
\hline \multicolumn{6}{|l|}{1994} \\
\hline March & 8.1 & 8.7 & 9.1 & 9.2 & 9.3 \\
\hline June & 8.1 & 8.7 & 9.1 & 9.2 & 9.3 \\
\hline September & 8.1 & 8.7 & 9.1 & 9.2 & 9.3 \\
\hline December & 8.9 & 9.5 & 10.1 & 10.5 & 12.3 \\
\hline \multicolumn{6}{|l|}{1995} \\
\hline March & 10.3 & 10.9 & 12.1 & 12.7 & 13.6 \\
\hline June & 11.0 & 11.5 & 12.8 & 13.2 & 14.5 \\
\hline September & 11.0 & 11.5 & 12.8 & 13.2 & 14.5 \\
\hline December & 11.0 & 11.5 & 12.8 & 13.2 & 14.5 \\
\hline \multicolumn{6}{|l|}{1996} \\
\hline March & 11.0 & 11.5 & 12.8 & 13.2 & 14.5 \\
\hline June & 13.8 & 14.2 & 15.3 & 15.5 & 15.4 \\
\hline September & 13.6 & 13.9 & 14.1 & 14.1 & 14.0 \\
\hline December & 15.0 & 15.3 & 15.5 & 15.4 & 15.2 \\
\hline \multicolumn{6}{|l|}{1997} \\
\hline March & 15.0 & 15.3 & 15.5 & 15.4 & 15.2 \\
\hline June & 13.6 & 14.2 & 14.5 & 14.5 & 14.5 \\
\hline September & 13.6 & 14.0 & 13.9 & 13.9 & 13.9 \\
\hline December & 13.2 & 13.6 & 13.7 & 13.5 & 13.5 \\
\hline \multicolumn{6}{|l|}{1998} \\
\hline March & 12.6 & 12.9 & 12.9 & 12.8 & 12.7 \\
\hline June & 14.2 & 16.8 & 16.9 & 15.0 & 14.5 \\
\hline September & 19.3 & 19.7 & 20.5 & 20.6 & 20.6 \\
\hline Decenber & 16.0 & 16.3 & 16.3 & 16.2 & 15.8 \\
\hline \multicolumn{6}{|l|}{1999} \\
\hline March & 13.1 & 13.2 & 13.1 & 12.7 & 13.9 \\
\hline June & 11.4 & 11.7 & 11.7 & 11.7 & 12.0 \\
\hline September & 9.4 & 9.7 & 9.7 & 10.1 & 10.5 \\
\hline December & 8.9 & 9.3 & 9.6 & 9.7 & 10.1 \\
\hline \multicolumn{6}{|l|}{2000} \\
\hline March & 7.9 & 8.3 & 8.5 & 8.7 & 9.6 \\
\hline June & 7.9 & 8.6 & 8.9 & 9.3 & 10.5 \\
\hline September & 8.0 & 8.7 & 8.8 & 9.0 & 9.5 \\
\hline
\end{tabular}


Table 19. Lesotho: Interest Rates at Commercial Banks, March 1995-Septernber 2000

(In percent per annusn; end of period)

\begin{tabular}{|c|c|c|c|c|c|c|c|c|c|c|c|c|c|c|c|c|c|c|c|c|c|c|c|}
\hline & \multicolumn{4}{|c|}{1995} & \multicolumn{4}{|c|}{1996} & \multicolumn{4}{|c|}{1997} & \multicolumn{4}{|c|}{1998} & \multicolumn{4}{|c|}{1999} & \multicolumn{3}{|c|}{2000} \\
\hline & March & June & Sep. & $\overline{\text { Dec. }}$ & March & June & Sep. & Dec. & March & June & Sep. & $\overline{D e c}$. & March & Jutre & Sep. & $\overline{\text { Dex. }}$ & March & June & Sep. & $\overline{\text { Dec. }}$ & March & June & Sep. \\
\hline \multicolumn{24}{|l|}{$\begin{array}{l}\text { Lending rates } 1 / \\
\text { Minimum }\end{array}$} \\
\hline Minimum & 16.5 & 16.5 & 16.5 & 16.5 & 16.5 & 16.5 & 18.0 & 18.5 & 18.5 & 18.1 & 18.1 & 17.1 & 17.1 & 18.5 & 23.5 & 22.0 & 19.6 & 19.3 & 18.5 & 17.7 & 17.0 & 17.0 & 17.0 \\
\hline Maximum & 26.5 & 26.5 & 26.5 & 26.5 & 26.5 & 29.0 & 28.0 & 28.5 & 28.1 & 28.1 & 28.1 & 28.1 & 27.1 & 28.5 & 33.5 & 32.0 & 25.3 & 29.3 & 29.0 & 28.0 & 27.0 & 25.3 & 24.5 \\
\hline \multicolumn{24}{|l|}{$\begin{array}{l}\text { Deposit rates } \\
\text { Savings deposits } 2\end{array}$} \\
\hline $\begin{array}{l}\text { Savings deposits } 2 / \\
\text { Time deposits }\end{array}$ & 6.1 & 6.2 & 6.2 & 6.2 & 6.2 & 6.0 & 6.0 & 6.0 & 6.0 & 6.0 & 6.0 & 6.0 & 6.0 & 6.0 & 6.0 & 5.5 & 4.0 & 3.0 & 3.0 & 3.5 & 3.5 & 3.5 & 3.5 \\
\hline 31 days & 10.3 & 10.3 & 10.3 & 10.3 & \multicolumn{18}{|c|}{$\begin{array}{l}\text { Time deposits } \\
31 \text { days }\end{array}$} & \\
\hline 1 year & 13.6 & 13.6 & 13.6 & 13.6 & 13.6 & 13.8 & 11.5 & 11.3 & 11.3 & 10.6 & $\begin{array}{r}8.8 \\
10.5\end{array}$ & $\begin{array}{r}0.0 \\
10.5\end{array}$ & $\begin{array}{r}8.8 \\
10.5\end{array}$ & $\begin{array}{r}9.6 \\
10.5\end{array}$ & 120 & 11.0 & 6.0 & 6.0 & 6.0 & 4.0 & 4.0 & 4.0 & 4.0 \\
\hline \multirow{2}{*}{\multicolumn{24}{|c|}{$\begin{array}{l}\text { Memorandumn items: } \\
\text { South African rates }\end{array}$}} \\
\hline & & & & & & & & & & & & & & & & & & & & & & & \\
\hline \multirow{2}{*}{\multicolumn{20}{|c|}{$\begin{array}{l}\text { Deposit rates } \\
\text { Notice (31 days) }\end{array}$}} & 15.5 & 14.5 & 14.5 & 14.5 \\
\hline Notice (31 days) & 12.5 & 13.0 & 13.5 & 14.3 & & & & & & & & & & & & & & & & & & & \\
\hline Fixed (12 months) & 14.3 & 15.0 & 14.5 & 13.9 & 13.5 & $\begin{array}{l}13.8 \\
15.1\end{array}$ & 13.3 & 17.0 & 16.3 & 15.5 & 15.5 & 14.5 & 13.0 & 18.0 & 20.9 & 17.8 & 14.8 & 13.0 & 11.0 & 10.3 & 9.5 & 9.3 & 9.3 \\
\hline & & & & & & & 84.9 & 10.0 & 15.4 & 14.8 & 14.1 & 14.0 & 12.6 & 15.8 & 21.0 & 17.4 & 13.7 & 12.8 & 11.4 & 11.4 & 10.5 & 10.5 & 9.8 \\
\hline
\end{tabular}

1/ Minimum and maximum lending rates are not statutory rates; they simply indicate the range of interest rates reported by banks.

2/ Minimum deposit rates; from December 1999, they are maximum deposit rates. 
Table 20. Lesotho: Comparative Money Market Rates, March 1994-September 2000 (In percent per annum; end of period)

\begin{tabular}{|c|c|c|c|c|}
\hline & \multicolumn{2}{|c|}{ Discount Rate } & \multicolumn{2}{|c|}{ Treasury Bills } \\
\hline & CBL $1 /$ & SARB 2/ & Lesotho & South Africa \\
\hline \multicolumn{5}{|l|}{1994} \\
\hline March & 13.5 & 12.0 & 9.9 & 10.2 \\
\hline June & 13.5 & 12.0 & 9.3 & 10.7 \\
\hline September & 13.5 & 13.0 & 9.3 & 10.9 \\
\hline December & 13.5 & 13.0 & 10.4 & 12.7 \\
\hline \multicolumn{5}{|l|}{1995} \\
\hline March & 13.5 & 14,0 & 10.4 & 12.8 \\
\hline June & 15.5 & 15.0 & 12.3 & 14.1 \\
\hline September & 15.5 & 15.0 & 13.0 & 14.0 \\
\hline December & 15.8 & 15.0 & 13.0 & 14.2 \\
\hline \multicolumn{5}{|l|}{1996} \\
\hline March & 15.8 & 15.0 & 13.0 & 14.2 \\
\hline June & 15.8 & 16.0 & 15.5 & 15.7 \\
\hline September & 16.0 & 16.0 & 14.3 & 15.1 \\
\hline December & 17.0 & 17.0 & 14.3 & 16.1 \\
\hline \multicolumn{5}{|l|}{1997} \\
\hline March & 17.0 & 17.0 & 15.7 & 15.8 \\
\hline June & 16.0 & 17.0 & 15.2 & 15.2 \\
\hline September & 16.6 & 17.0 & 14.2 & 14.7 \\
\hline December & 15.6 & 16.0 & 13.9 & 14.7 \\
\hline \multicolumn{5}{|l|}{1998} \\
\hline March & 15.6 & 16.0 & 13.1 & 12.9 \\
\hline June & 17.0 & 16.0 & 17.2 & 18.8 \\
\hline September & 21.0 & 21.9 & 20.7 & 20.1 \\
\hline December & 19.5 & 19.3 & 16.6 & 17.0 \\
\hline \multicolumn{5}{|l|}{1999} \\
\hline March & 19.5 & 16.5 & 15.5 & 14.4 \\
\hline June & 19.0 & 15.5 & 12.6 & 12.9 \\
\hline September & 19.0 & 12.6 & 10.5 & 10.8 \\
\hline December & 19.0 & 12.0 & 9.9 & 10.7 \\
\hline \multicolumn{5}{|l|}{2000} \\
\hline March & 19.0 & 11.8 & 9.1 & 9.8 \\
\hline June & 19.0 & 11.8 & 9.1 & 10.4 \\
\hline September & 19.0 & 11.8 & 9.1 & 10.2 \\
\hline \multicolumn{5}{|c|}{ Source: Central Bank of Lesotho. } \\
\hline $\begin{array}{l}1 / \text { Central B } \\
2 / \text { South Afr }\end{array}$ & $\begin{array}{l}10 . \\
\text { Bank. }\end{array}$ & & & \\
\hline
\end{tabular}


Table 21. Lesotho: Balance of Payments, 1995/96-1999/2000 1/

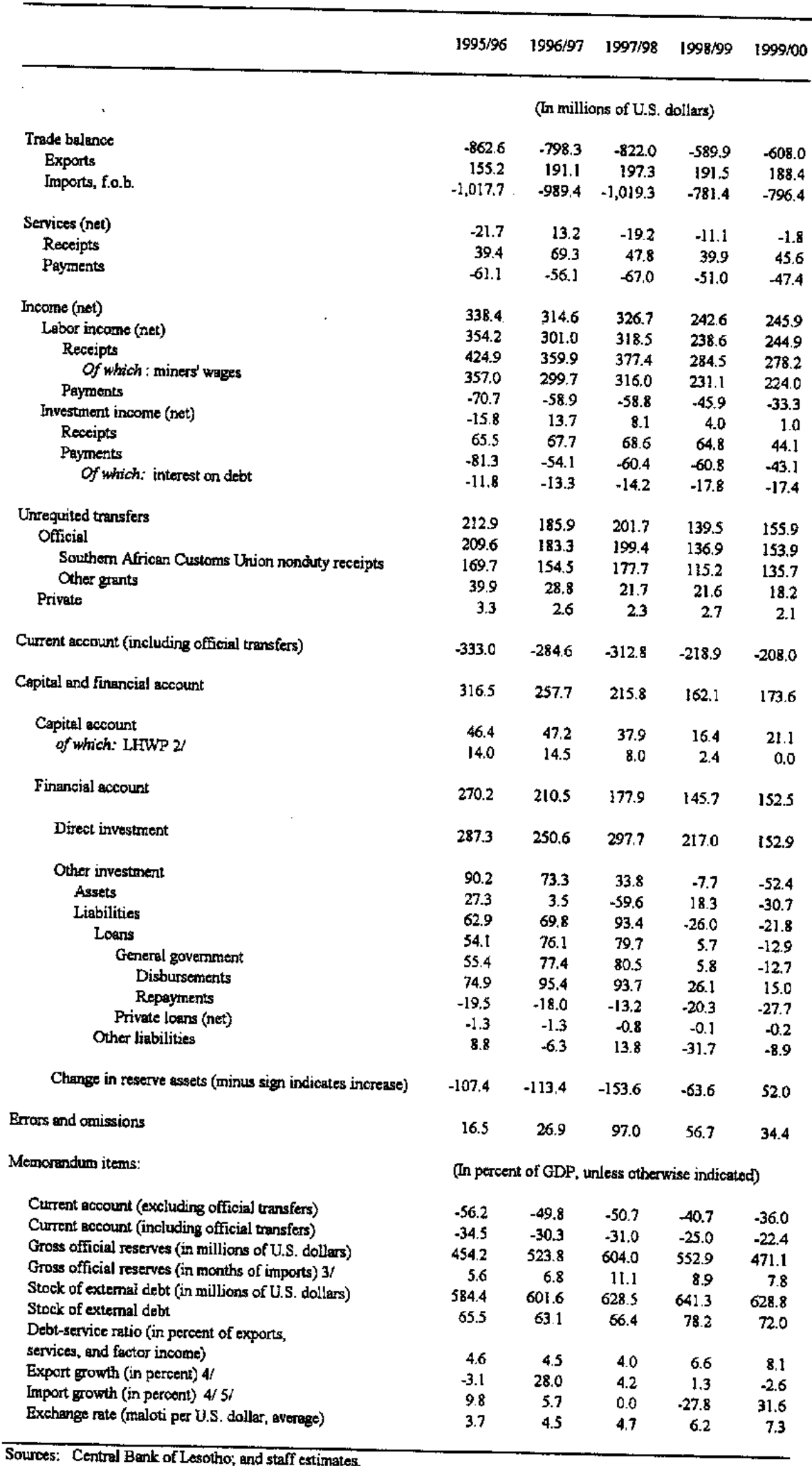

1/ Fiscal year beginning in April.

$2 /$ Lesotho Highlands Water Project (LHWP).

3/ Bosed on next year's imports and excludes LHWP.

4/ In real terms.

5/ Excludes LHWP imports. 
Table 22. Lesotho: Balance of Payments, 1995/96 - 1999/2000 d/

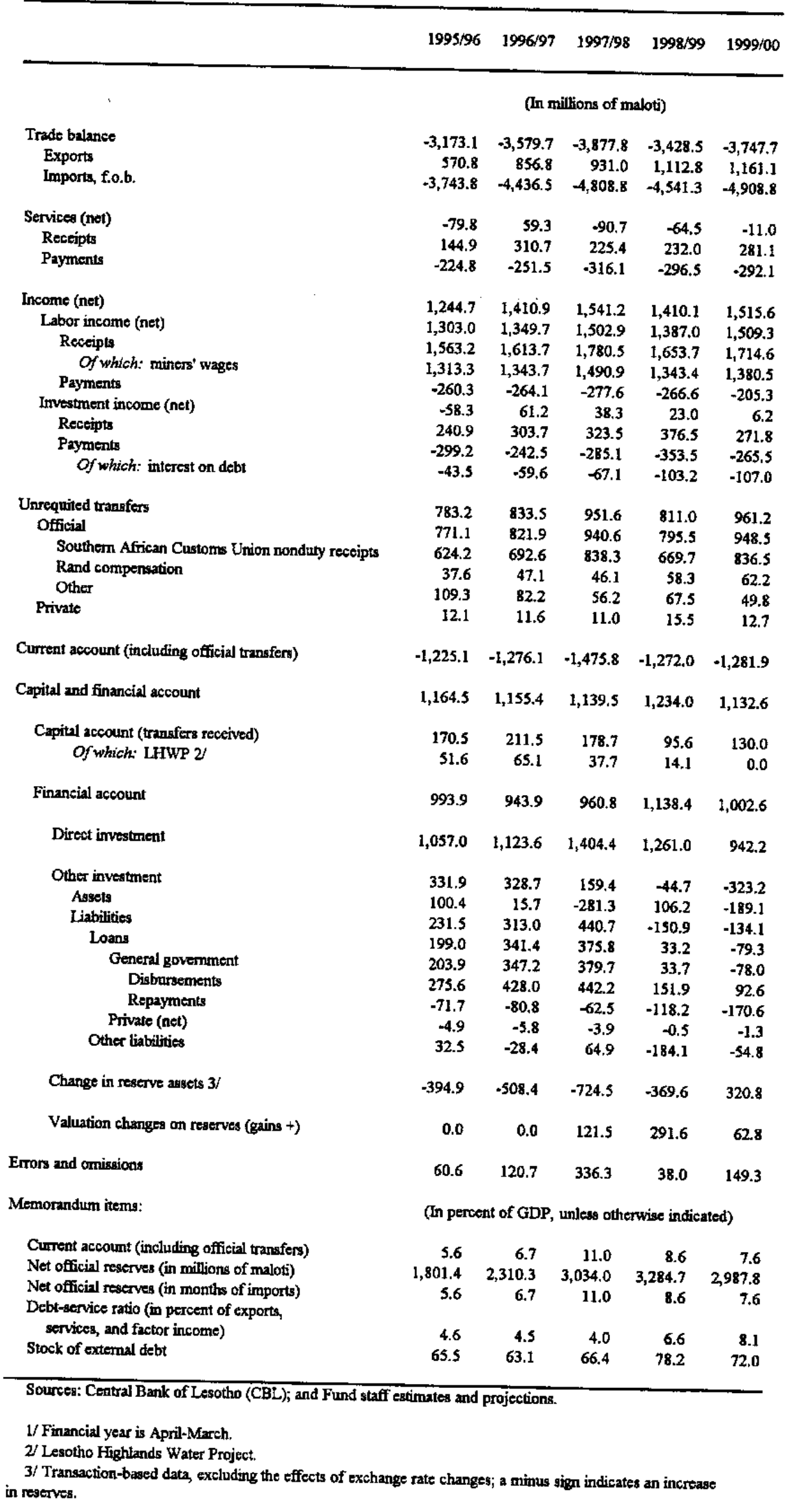


Table 23. Lesotho: Services and Income Account, 1995/96-1999/2000 1/

(In millions of maloti)

\begin{tabular}{|c|c|c|c|c|c|}
\hline & $1995 / 96$ & $1996 / 97$ & $1997 / 98$ & $1998 / 99$ & $1999 / 00$ \\
\hline Nonfactor services (net) & -79.8 & 59.3 & -90.7 & -64.5 & -11.0 \\
\hline Credit & 144.9 & 310.7 & 225.4 & 232.0 & 281.1 \\
\hline Shipment & 0.0 & 0.0 & 0.0 & 0.0 & 0.0 \\
\hline Other transportation & 8.3 & 7.5 & 11.9 & 5.7 & 3.9 \\
\hline Travel & 102.5 & 114.2 & 104.5 & 109.2 & 154.9 \\
\hline Sales of water (and power) & 0.0 & 152.2 & 67.3 & 76.6 & 79.9 \\
\hline Other official & 32.9 & 35.9 & 37.1 & 40.8 & 44.3 \\
\hline Other private & 1.3 & 0.9 & 4.6 & -0.3 & -1.9 \\
\hline Debit & -224.8 & -251.5 & -316.1 & -296.5 & -292.1 \\
\hline Shipment & -147.9 & -167.6 & -186.8 & -175.8 & -190.3 \\
\hline Other transportation & -16.3 & -17.7 & -16.2 & -17.0 & -21.0 \\
\hline Travel & -48.4 & -58.9 & -61.9 & -83.2 & -69.3 \\
\hline Other official & -8.5 & -3.3 & -43.6 & -16.4 & -12.3 \\
\hline Other private & -3.6 & -4.0 & -7.6 & -4.1 & 0.7 \\
\hline Factor incomes (net) & $1,244.7$ & $1,410.9$ & $1,541.2$ & $1,410.1$ & $1,515.6$ \\
\hline Credit & $1,804.1$ & $1,917.4$ & $2,103.9$ & $2,030.2$ & $1,986.4$ \\
\hline Investment income & 240.9 & 303.7 & 323.5 & 376.5 & 271.8 \\
\hline Interest earned by commercial banks & 179.0 & 242.6 & 295.5 & 348.7 & 242.9 \\
\hline Interest earned by the central bank & 61.8 & 61.1 & 27.9 & 27.9 & 28.9 \\
\hline Labor income & $1,563.2$ & $1,613.7$ & $1,780.5$ & $1,653.7$ & $1,714.6$ \\
\hline Debit & -559.4 & -506.6 & -562.7 & -620.1 & -470.8 \\
\hline Investment income & -299.2 & -242.5 & -285.1 & -353.5 & -265.5 \\
\hline Dividends and profits & -255.6 & -183.0 & -218.1 & -250.3 & -158.6 \\
\hline Interest & -43.5 & -59.6 & -67.1 & -103.2 & -107.0 \\
\hline Labor income & -260.3 & -264.1 & -277.6 & -266.6 & -205.3 \\
\hline Total services and income (net) & $1,164.8$ & $1,470.1$ & $1,450.5$ & $1,345,6$ & $1,504.6$ \\
\hline
\end{tabular}

Source: Central Bank of Lesotho.

1/ Financial year is April-March. 
Table 24. Lesotho: Lesotho Miners in South Africa, 1995-99

\begin{tabular}{|c|c|c|c|c|c|}
\hline & 1995 & 1996 & 1997 & 1998 & 1999 \\
\hline Total average number employed (in thousands) & 103.7 & 101.7 & 95.9 & 80.9 & 68.6 \\
\hline Annual percentage change & 2.7 & -1.9 & -5.7 & -15.7 & -15.2 \\
\hline \multicolumn{6}{|l|}{ Of which: employed through TEBA $1 /$} \\
\hline Average number (in thousands) & 87.8 & 85.7 & 78.8 & 66.2 & 55.4 \\
\hline Annual percentage change & 11.9 & -2.4 & -7.9 & -16.1 & -16.4 \\
\hline Employed through TEBA/total employed (in percent) & 84.6 & 84.2 & 82.2 & 81.8 & 80.7 \\
\hline Average annual earnings (in maloti) $2 /$ & $16,801.0$ & $19,186.0$ & $21,193.0$ & $24,678.0$ & $27,657.0$ \\
\hline Anmual percentage change & 15.4 & 14.2 & 10.5 & 16.4 & 12.1 \\
\hline Total earnings (in millions of maloti) & $1,743.0$ & $1,951.9$ & $2,032.7$ & $1,996.2$ & $1,897.4$ \\
\hline Annual percentage change & 18.5 & 12.0 & 4.1 & -1.8 & -4.9 \\
\hline Miners' remittances (in millions of maloti) $3 /$ & $1,242.8$ & $1,391.7$ & $1,321.2$ & $1,217.7$ & $1,157.4$ \\
\hline Miners' remittances (as percentage of total eamings) & 71.3 & 71.3 & 65.0 & 61.0 & 61.0 \\
\hline Miners' remittances (annual percentage change) & 18.5 & 12.0 & -5.1 & -7.8 & -4.9 \\
\hline
\end{tabular}

Sources: Central Bank of Lesotho; Department of Labor; and the Employment Bureau for Africa.

1/ The Employment Bureau for Africa, an agency of the South African Chamber of Mines.

2/ Average for Lesotho miners, including overtime payments and repatriation allowances, as reported by the South African Chamber of Mines.

3/ Estimated by the Central Bank of Lesotho as approximately 71 percent of total earnings, except for 1997 and 1998, which are based on incomplete data of the Department of Labor published in the the Central Bank's Quarterly Review. 
Table 25. Lesotho: Composition of Recorded Exports, 1995-99

\begin{tabular}{|c|c|c|c|c|c|}
\hline & 1995 & 1996 & 1997 & 1998 & 1999 \\
\hline & \multicolumn{5}{|c|}{ (In millions of maloti, unless otherwise indicated) } \\
\hline Foodstuffs, etc. & 31.2 & 33.8 & 43.8 & 75.5 & 119.3 \\
\hline Cereals & 7.2 & 9.2 & 18 & 23.8 & 36.2 \\
\hline \multicolumn{6}{|l|}{ Beans, peas, and } \\
\hline other vegetables & 2.5 & 1.3 & 4.9 & 4.9 & 2.3 \\
\hline Animal feed & 8.4 & 8.4 & 15.7 & 4.2 & 2.3 \\
\hline Beverages and tobacco & 1.6 & 0.5 & 0.1 & 38.7 & 73.4 \\
\hline Other foodstuffs & 11.5 & 14.4 & 5.1 & 3.9 & 5.1 \\
\hline Live animals & 3.8 & 1.6 & 2.3 & 7.0 & 6.5 \\
\hline Cattle & 2.7 & 1.6 & 2.3 & 3.1 & 3.0 \\
\hline Sheep and goats & 1.1 & $\ldots$ & $\ldots$ & $\ldots$ & $\ldots$ \\
\hline Poultry & $\cdots$ & $\cdots$ & $\cdots$ & 3.9 & 3.5 \\
\hline Livestock materials & 34.6 & 32.9 & 29.2 & 18.8 & 15.6 \\
\hline Wool & 27.4 & 22.7 & 23.5 & 16.7 & 14.6 \\
\hline Mohair & 5.2 & 9.4 & 4.9 & 1.2 & 0.9 \\
\hline Hides and skins & 1.5 & 0.7 & 0.8 & 0.9 & 0.1 \\
\hline Crude materials & 0.5 & 0.1 & 0.0 & 0.0 & 0.0 \\
\hline Diamonds & 1.6 & 1.6 & 1.3 & 0.4 & 0.7 \\
\hline Manufactures & 509.2 & 741.7 & 826.6 & 857.9 & 631.7 \\
\hline Chemicals and petroleum & 7.4 & 24.7 & 16.6 & 6.6 & 5.8 \\
\hline Leather products & 0.0 & 0.3 & 0.0 & 0.0 & 0.2 \\
\hline Wood products & 0.0 & 0.3 & 0.0 & 0.5 & 0.1 \\
\hline Yarn and textiles, etc. & 4.3 & 3.2 & 6.3 & 5.6 & 2.4 \\
\hline Road vehicles & 34.3 & 18.5 & 32.7 & 15.5 & 4.8 \\
\hline Furniture and parts & 10.1 & 10.8 & 11.3 & 4.9 & 5.9 \\
\hline Clothing, etc. & 284.1 & 368.8 & 403.4 & 415.4 & 612.5 \\
\hline Footwear & 44.7 & 153.1 & 177.4 & 253.6 & $\ldots$ \\
\hline Other manufactures & 124.3 & 162.0 & 178.9 & 155.8 & $\ldots$ \\
\hline Unclassified & 0.2 & 0.5 & 0.7 & 150.1 & 280.3 \\
\hline \multirow{3}{*}{$\begin{array}{l}\text { Total value } \\
\text { Change (in percent) }\end{array}$} & 580.6 & 812.1 & 903.9 & $1,109.7$ & $1,054.1$ \\
\hline & 14.0 & 39.9 & 11.3 & 22.8 & -5.0 \\
\hline & \multicolumn{5}{|c|}{ (Volume as indicated) } \\
\hline Wool (in metric tons) & $1,957.8$ & $2,082.5$ & $1,861.3$ & 950.8 & $\ldots$ \\
\hline Mohair (in metric tons) & 142.3 & 373.7 & 258.5 & $1,999.2$ & $\ldots$ \\
\hline Diamonds (in thousands of carats) & 13.9 & 1.2 & 0.6 & 0.4 & $\ldots$ \\
\hline Value per carat (in maloti) & 115.0 & 169.5 & 136.1 & 164.1 & $\ldots$ \\
\hline
\end{tabular}


Table 26. Lesotho: Direction of Trade, 1995 - 99 1/

(In millions of maioti)

\begin{tabular}{|c|c|c|c|c|c|c|c|c|c|c|}
\hline & \multicolumn{2}{|c|}{1995} & \multicolumn{2}{|c|}{1996} & \multicolumn{2}{|c|}{1997} & \multicolumn{2}{|c|}{1998} & \multicolumn{2}{|c|}{1999} \\
\hline & Imports & Exports & Imports & Exports & Imposts & Exports & Imports & Exports & Imports & Exports \\
\hline World & $3,994.7$ & 580.6 & $4,815.6$ & 812.1 & $5,253.5$ & 902.5 & $4,915.7$ & 998.7 & $2,868.5$ & $1,054.2$ \\
\hline Africa & $3,623.7$ & 306.6 & $4 ; 462.3$ & 569.6 & $4,704.4$ & 581.4 & $4,436.1$ & 654.9 & $2,327.6$ & 556.0 \\
\hline Common customs area & $3,621.3$ & 299.6 & $4,417.7$ & 562.6 & $4,687.1$ & 580.0 & $4,408.0$ & 652.9 & $2,324.6$ & 554.4 \\
\hline Other Africa & 2.4 & 7.0 & 44.6 & 7.0 & 17.3 & 1.4 & 28.1 & 2.0 & 3.0 & 1.6 \\
\hline European Union & 84.2 & 53.8 & 55.4 & 43.6 & 87.5 & 11.7 & 40.5 & 7.4 & 80.3 & 2.0 \\
\hline Belgium & 1.6 & $\ldots$ & 1.0 & $\ldots$ & 7.5 & $\ldots$ & 2.9 & $\ldots$ & 4.0 & 0.1 \\
\hline Denmark & 2.5 & $\ldots$ & 0.9 & $\ldots$ & 0.4 & $\ldots$ & 0.9 & $\ldots$ & 1.5 & $\ldots$ \\
\hline France & 36.7 & $\ldots$ & 16.2 & $\ldots$ & 27.9 & $\ldots$ & 3.2 & $\ldots$ & 4.3 & 1.0 \\
\hline Germany & 19.6 & $\ldots$ & 18.3 & $\ldots$ & 4.0 & $\ldots$ & 2.2 & $\ldots$ & 23.7 & $\ldots$ \\
\hline Italy & 7.9 & $\ldots$ & 3.5 & $\ldots$ & 2.7 & $\ldots$ & 2.8 & $\ldots$ & 17.5 & $\ldots$ \\
\hline Netherlands & 0.7 & $\ldots$ & 1.0 & $\ldots$ & 2.0 & $\ldots$ & 5.5 & $\ldots$ & 1.7 & $\ldots$ \\
\hline United Kingdom & 15.2 & $\ldots$ & 14.5 & $\cdots$ & 43.1 & $\ldots$ & 23.1 & $\ldots$ & 27.6 & 0.5 \\
\hline Other Europe & 36.2 & 0.5 & 19.4 & 0.1 & 11.4 & 0.3 & 26.9 & 0.2 & 17.5 & 0.4 \\
\hline North America $2 /$ & 29.0 & 218.3 & 23.7 & 198.5 & 52.5 & 308.4 & 84.2 & 333.1 & 39.8 & 495.0 \\
\hline Canada & 13.9 & $\ldots$ & 9.5 & $\ldots$ & 33.9 & $\ldots$ & 49.5 & $\ldots$ & 34.4 & 3.1 \\
\hline United States & 15.1 & $\ldots$ & 14.2 & $\cdots$ & 18.6 & $\cdots$ & 34.7 & $\ldots$ & 5.4 & 492.0 \\
\hline Asia & 219.5 & 1.4 & 254.0 & 0.3 & 394.8 & 0.7 & 309.2 & 2.2 & 372.4 & 0.2 \\
\hline Japan & 21.0 & $\ldots$ & 24.6 & $\ldots$ & 24.7 & $\ldots$ & 21.0 & $\ldots$ & 23.3 & $\ldots$ \\
\hline Hong Kong S.A.R. & 30.1 & $\ldots$ & 8.2 & $\ldots$ & 22.6 & $\ldots$ & 19.2 & $\ldots$ & 31.0 & $\ldots$ \\
\hline Taiwan Province of China & 114.8 & $\ldots$ & 154.5 & $\ldots$ & 192.1 & $\ldots$ & 183.5 & $\ldots$ & 195.2 & $\ldots$ \\
\hline Other & 53.6 & $\ldots$ & 66.7 & $\ldots$ & 155.4 & $\cdots$ & 85.6 & $\ldots$ & 122.9 & 0.2 \\
\hline Oceania & 1.0 & 0.0 & 0.8 & 0.0 & 2.9 & 0.0 & 18.9 & 0.9 & 30.9 & 0.5 \\
\hline
\end{tabular}

1/ Imports are c.i.f., duty exclusive, and exchuding donated food; exports are fo.b.

2/ Almost all of these exports are to the United States. 
Table 27. Lesotho: Public and Publicly Guaranteed External Debt Outstanding, 1995/96-1999/2000 1/

\begin{tabular}{|c|c|c|c|c|c|}
\hline ' & $1995 / 96$ & $1996 / 97$ & $1997 / 98$ & $1998 / 99$ & $1999 / 00$ \\
\hline & \multicolumn{5}{|c|}{ (In millions of U.S. dollars) } \\
\hline Multilateral sources & 493.5 & 498.9 & 499.5 & 502.6 & 491.2 \\
\hline IMF & 37.1 & 32.0 & 26.1 & 19.7 & 14.8 \\
\hline World Bank Group & 209.1 & 218.3 & 222.6 & 233.2 & 236.3 \\
\hline IBRD & 53,8 & 59.2 & 56.9 & 51.9 & 49.3 \\
\hline International Development Association & 155.3 & 159.0 & 165.7 & 181.3 & 187.1 \\
\hline African Development Bank & 23.8 & 18.3 & 15.2 & 13.6 & 12.5 \\
\hline African Development Fund & 148.6 & 149.8 & 150.3 & 153.8 & 154.0 \\
\hline Other & 74.9 & 80.5 & 85.3 & 82.3 & 73.5 \\
\hline Bilateral sources & 57.0 & 73.2 & 73.6 & 99.4 & 102.3 \\
\hline Commercial & 53.0 & 60.7 & 84.2 & 90.0 & 88.0 \\
\hline Commercial banks & 41.5 & 49.4 & 65.5 & 66.4 & 66.2 \\
\hline Export credits & 11.4 & 11.3 & 18.7 & 23.6 & 21.8 \\
\hline \multirow[t]{2}{*}{ Total } & 603.5 & 632.7 & 657.3 & 691.9 & 681.4 \\
\hline & \multicolumn{5}{|c|}{ (As percent of total debt, unless otherwise indicated) } \\
\hline Multilateral & 81.8 & 78.8 & 76.0 & 72.6 & 72.1 \\
\hline Bilateral & 9.4 & 11.6 & 11.2 & 14.4 & 15.0 \\
\hline Commercial & 8.8 & 9.6 & 12.8 & 13.0 & 12.9 \\
\hline & \multicolumn{5}{|c|}{ (As percent of GDP) $2 /$} \\
\hline Multilateral & 55.6 & 52.2 & 52.7 & 61.6 & 55.3 \\
\hline Bilateral & 6.4 & 7.7 & 7,8 & 12.2 & 11.5 \\
\hline Commercial & 6.0 & 6.3 & 8.9 & 11.0 & 9.9 \\
\hline Total & 68.0 & 66.2 & 69.4 & 84.8 & 76.8 \\
\hline \multicolumn{6}{|l|}{ Memorandum items: } \\
\hline External debt/GDP ratio (in percent) & 68.0 & 66.2 & 69.4 & 84.8 & 76.8 \\
\hline GDP (in millions of maloti) & $3,549.9$ & $4,216.3$ & $4,764.2$ & $5,078.8$ & $5,730.9$ \\
\hline Maloti per U.S. dollar (end of fiscal year) & 4.0 & 4.4 & 5.0 & 6.2 & 6.5 \\
\hline
\end{tabular}


Lesotho: Summary of the Tax System, February 14, 2001

(All amounts in maloti)

\begin{tabular}{cc} 
Tax \\
\hline $1 . \quad$ Taxes on net income and profits \\
$1.1 \quad \begin{array}{l}\text { Taxes on companies, corporations, } \\
\text { or enterprises }\end{array}$
\end{tabular}

\subsubsection{Income tax}

Income Tax Act 1993,

Order No. 9 of 1993 (this act

Act);

Income Tax (Amendment)

Act 1994; and Income Tax

(Amendment) Act 1996.

1.1.2 Gambling levy

The Casino Act, No. 26 of

1969; Legal Order No. 42 of

1971. Casino Order No. 4 of

1989. income from all geographical

sources (in the case of resident

companies) including on

specified fringe benefits to

employees. Dividends paid by

a resident company to a

resident shareholder are

excmpt but advance corporate

tax applies.

Deductions include normal

operating costs, expenditures

for repair and maintenance,

and depreciation of plant and

machinery. Expenditure on the

training of Basotho workers is

deductible up to 125 percent of actual expenditure incurred.

The incorne of pension funds,

life insurance companies, and charitable institutions is

exempt.

A levy on gross profits of

gambling casinos.
For all sectors of activity other than manufacturing, 35 percent a reduced rate ( 15 percent) is applicable to all manufacturing companies other than those approved for Pioneering

Industries benefits before

August 3, 1990 (see Item 6 below).

For nonresident companies, 25 percent.

15 percent 


\section{Lesotho: Summary of the Tax System, February 14, 2001}

(All amounts in maloti)

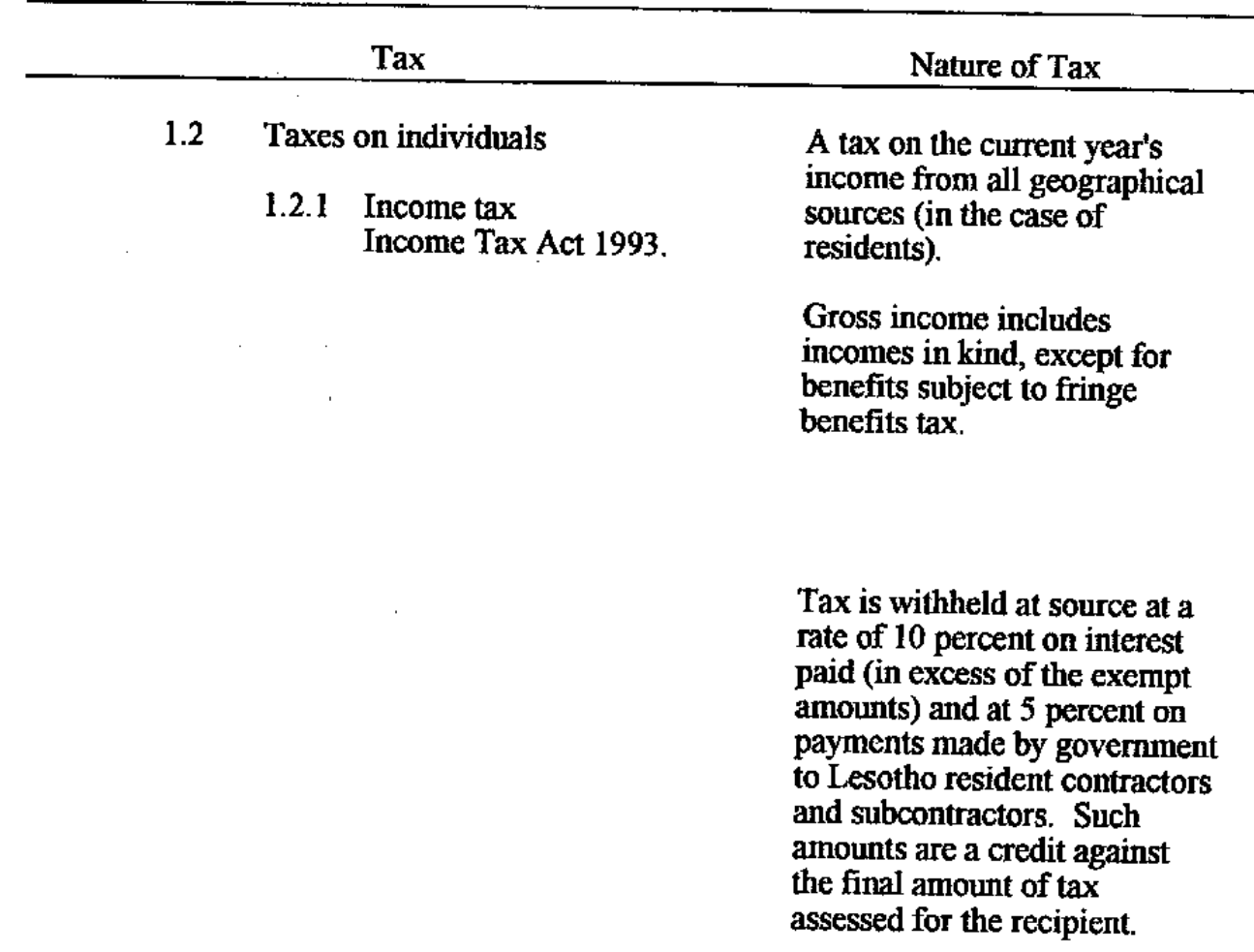

1.2.2 Withholding tax Income Tax Act 1993.

\section{A tax on income from dividends, interest, royalties, natural resource payments, management charges, or services contracts earned within Lesotho by nonresidents.}

Exemptions and Deductions

Rates

Exempt incomes include: the first M 500 of interest from savings;

income from subsistence

farming;

scholarships; and foreign-

source property income of expatriate taxpayers.

Deductions include expenses of deriving income.

A uniform personal tax credit of $M 2,640$ per taxpayer was introduced in April 1996.

Residents marginal rate (\%)

First $M 30,000 \quad 25$ percent Over M 30,000 35 percent

Nonresidents marginal rate (\%)

All chargeable

Icome

25 percent

Dividends from manufacturing companies are exempt.

Withholding tax is 25 percent.

For royalties from nonmanufacturing companies, 15 percent.

On service contracts earned within Lesotho by nonresidents. 10 percent 
Lesotho: Summary of the Tax System, February 14, 2001

(All amounts in maloti)

\begin{tabular}{|c|c|c|c|c|c|}
\hline & & Tax & Nature of Tax & Exemptions and Deductions & Rates \\
\hline & & $\begin{array}{l}\text { 1.2.3. Income Tax (Amendment } \\
\text { Act, 1999) }\end{array}$ & A tax on farm income & & Rated at $15 \%$ \\
\hline & & $\begin{array}{l}\text { 1.2.4. Income Tax (Amendment } \\
\text { Act, 2000) }\end{array}$ & $\begin{array}{l}\text { Taxation of LHDA activities in } \\
\text { terms of Protocol V to the } \\
\text { LHDA Treaty }\end{array}$ & $\begin{array}{l}\text { Exemptions as specified in } \\
\text { Protocol V to the LHDA } \\
\text { Treaty }\end{array}$ & $\begin{array}{l}\text { Differential rates applied to } \\
\text { companies contracted by LHDA }\end{array}$ \\
\hline \multirow[t]{5}{*}{2.} & \multicolumn{5}{|c|}{ Taxes on property } \\
\hline & \multirow[t]{2}{*}{2.1} & Property rates & & & \\
\hline & & $\begin{array}{l}\text { Valuation and Rating Act 1980; } \\
\text { Urban Government Act 1993; and } \\
\text { Legal Notice No. } 10 \text { of } 1997 .\end{array}$ & $\begin{array}{l}\text { Taxes on urban land and } \\
\text { improvements based on capital } \\
\text { value of property, as assessed } \\
\text { periodically. Improvements } \\
\text { valued on basis of depreciated } \\
\text { replacement value. }\end{array}$ & $\begin{array}{l}\text { These taxes are currently } \\
\text { applied only within Maseru, } \\
\text { Teyateyaneng, and Mafeteng. } \\
\text { Government property is } \\
\text { subject to a grant in lieu. }\end{array}$ & $\begin{array}{l}\text { Rates of } 0.25 \text { percent on } \\
\text { residential property; } 2.0 \text { percent } \\
\text { on commercial property; and } \\
2.75 \text { percent on industrial } \\
\text { property. }\end{array}$ \\
\hline & \multirow[t]{2}{*}{2.2} & $\begin{array}{l}\text { Ground rents } \\
\text { Land Act No. } 17 \text { of } 1979\end{array}$ & & & \\
\hline & & $\begin{array}{l}\text { Land Regulations, Legal Notice No. } \\
15 \text { of } 1980 \text {; and Legal Notice No. } \\
\text { I31 of } 1991 \text {. }\end{array}$ & $\begin{array}{l}\text { A fee for use right to occupy } \\
\text { land. Charged according to } \\
\text { area of land and location. }\end{array}$ & $\begin{array}{l}\text { Owner-occupiers are } \\
\text { exempted. }\end{array}$ & $\begin{array}{l}\text { M } 0.05-0.10 \text { per annum per } \\
\text { square meter for residential } \\
\text { land; M } 0.25-0.30 \text { per anmum } \\
\text { per square meter for commer- } \\
\text { cial land. Levy of } 5 \text { percent for } \\
\text { late payment. }\end{array}$ \\
\hline
\end{tabular}


Lesotho: Summary of the Tax System, February 14, 2001

(All amounts in maloti)

\begin{tabular}{ccc} 
Tax & Nature of Tax $\quad$ Exemptions and Deductions \\
\hline
\end{tabular}

\subsection{Death taxcs}

\subsubsection{Estate duty}

Proclamation No. 20 of 1935 A duty paid by the estate in as amended. respect of property passing on the death of the person who owned the property at the time of death.

\subsubsection{Succession duty}

Proclamation No. 20 of 1935 as amended.
A duty levied on all successions accruing to any person.
Any amount received under an insurance policy is not subject to tax.

\section{Successions accruing to a} surviving spouse, to the Lesotho government, and to nonprofit public institutions within Lesotho are exempt.

\subsection{Transfer duty} Transfer Duty Act, 1965, No. 7 of 1966; Transfer Duty Order, 1972, Order No. 1 of 1972.
A duty levied on the transfer of immovable property (including lease contracts for at least ten years and any rights to minerals).
The following are exempt: the Lesotho government and its departments, the Lesotho Electricity Corporation, the Lesotho Bank, the Lesotho Airways Corporation, the Lesotho National Development Corporation; local authorities; nonprofit public institutions and public hospitals; and a surviving spouse for the estate of a deceased spouse.
Three ten thousandths $\mathrm{M} 2$ for every M 200 or part thereof, subject to a maximum rate of M 0.67 per M 2. A rebate of M 600 is deducted from the amount of duty calculated.

\section{A rate of duty varying} according to the degree of relationship of the successor from 3 percent to 12 percent of the dutiable amount. A

1 percent surcharge is levied on dutiable successions exceeding M 20,000.

A duty of 3 percent on the first $M 10,000$ of value and 4 percent on the excess value. 


\section{Lesotho: Summary of the Tax System, February 14, 2001}

(All amounts in maloti)

\begin{tabular}{|c|c|c|c|}
\hline $\operatorname{Tax}$ & Nature of Tax & Exemptions and Deductions & Rates \\
\hline $\begin{array}{l}\text { 3. Taxes on goods and services } \\
3.1 \quad \text { General Sales Tax } \\
\text { Sales Tax Act 1995, No. } 14 \text { of } 1995 \text {, published } \\
\text { April 29, 1996, implemented from August 31, } 1996 \\
\text { (this act repealed the Sales Tax Act } 1982 \text { except for } \\
\text { certain transitional administrative arrangements). }\end{array}$ & $\begin{array}{l}\text { A sales tax imposed on every } \\
\text { taxable supply in Lesotho and } \\
\text { on every import of goods and } \\
\text { services. The act provides for } \\
\text { the imposition of a single-stage } \\
\text { sales tax at retail level. The act } \\
\text { also paves the way for value- } \\
\text { added tax (VAT) in that it } \\
\text { provides for the claiming of } \\
\text { credit for import tax paid in } \\
\text { respect of certain supplies to, } \\
\text { or certain imports by, a taxable } \\
\text { vendor. It also provides for a } \\
\text { credit for certain taxes paid on } \\
\text { goods on hand at the time a } \\
\text { person becomes registered. } \\
\text { The act provides for mandatory } \\
\text { and voluntary applications by } \\
\text { vendors and compulsory } \\
\text { registration by the } \\
\text { Commissioner. Where the } \\
\text { vendor has been registered, the } \\
\text { Commissioner is obliged to } \\
\text { issue a sales tax registration } \\
\text { certificate. However, the } \\
\text { Commissioner may refuse to } \\
\text { issue a sales tax exemption } \\
\text { certificate to be registered } \\
\text { person. }\end{array}$ & $\begin{array}{l}\text { Under Section } 6(2) \text { the act } \\
\text { exempts from sales tax imports } \\
\text { of goods prescribed in } \\
\text { Schedule II (diplomatic } \\
\text { purchases, passengers' } \\
\text { baggage, household furniture } \\
\text { and effects of new rcsidents, } \\
\text { relief and supplies, temporary } \\
\text { imports, etc.). It further } \\
\text { exempts the goods acquired by } \\
\text { the vendor as raw materials for } \\
\text { use in mamufacturing or for re- } \\
\text { supply in substantially the } \\
\text { same state, and capital goods } \\
\text { supplied to, or imported by, a } \\
\text { manufacturer. } \\
\text { The following sales or supplies } \\
\text { are also exempted: goods as } \\
\text { part of the transfer of an } \\
\text { enterprise; goods to, or imports } \\
\text { by, an employee of a foreign } \\
\text { government or public } \\
\text { international organization } \\
\text { seconded } \\
\text { to Lesotho; water; public } \\
\text { postal services; passenger } \\
\text { transport by road or air; } \\
\text { medical and dental services; } \\
\text { specified financial services; } \\
\text { insurance services; and } \\
\text { educational services. } \\
\text { Government purchases are } \\
\text { exempt }\end{array}$ & $\begin{array}{l}\text { Rates are prescribed by the } \\
\text { minister by notice in the official } \\
\text { gazette. The existing rates are: } \\
\text { Goods Rate (in percent) } \\
\text { General } \\
\text { Liquor } 10 \\
\text { Telecommunications } \\
\text { And electricity } \\
\text { Exports }\end{array}$ \\
\hline
\end{tabular}


Lesotho: Summary of the Tax System, February 14, 2001

(All amounts in maloti)

\begin{tabular}{|c|c|c|c|c|}
\hline & Tax & Nature of Tax & Exemptions and Deductions & Rates \\
\hline 3.2 & $\begin{array}{l}\text { Excise taxes } \\
\text { Customs and Excise Consolidated } \\
\text { Act, No. } 10 \text { of } 1982 \text {. }\end{array}$ & $\begin{array}{l}\text { A tax on certain goods } \\
\text { manufactured and imported } \\
\text { into Lesotho, including beer, } \\
\text { spirits, wines matches, } \\
\text { tobacco, cigars, cigarettes, } \\
\text { petroleum oils, motor vehicles, } \\
\text { tractors, and electronic } \\
\text { products such as televisions. }\end{array}$ & $\begin{array}{l}\text { Exports and purchases by } \\
\text { charitable organizations are } \\
\text { exempt. }\end{array}$ & $\begin{array}{l}\text { Both specific and ad valorem } \\
\text { rates. }\end{array}$ \\
\hline 3.3. & $\begin{array}{l}\text { Trade licenses } \\
\text { Trading Enterprise Order, 1993; and } \\
\text { Order No. } 11 \text { of } 1997\end{array}$ & $\begin{array}{l}\text { Payable by traders carrying on } \\
\text { business. }\end{array}$ & $\begin{array}{l}\text { Charitable, religious, and } \\
\text { nonprofit institutions are } \\
\text { exempt. }\end{array}$ & $\begin{array}{l}\text { Ranging from M } 20 \text { to M } 500 \\
\text { depending on the type and size } \\
\text { of establishment. }\end{array}$ \\
\hline 3.4 & $\begin{array}{l}\text { Petrol levy } \\
\text { Fuel and Service Control Act 1983, } \\
\text { No. } 23 \text { of } 1983 \text {. Section 3(d) } \\
\text { empowers the Minister to impose } \\
\text { and collect a levy on fuel. Amended } \\
\text { by Legal Notice No. 63, August } \\
1988 \text {. }\end{array}$ & $\begin{array}{l}\text { A levy on petrol of all grades } \\
\text { or distillate supplied by any } \\
\text { person. }\end{array}$ & Paraffin (kerosene) is exempt. & $\begin{array}{l}\text { Rates are } 43 \text { lisente per liter on } \\
\text { petrol sold to public for private } \\
\text { cars, and } 37 \text { lisente per liter for } \\
\text { diesel used in industry, agri- } \\
\text { culture, and public buses. In } \\
\text { addition, there is an Equal- } \\
\text { ization Fund levy of } 3 \text { lisente } \\
\text { per liter. }\end{array}$ \\
\hline
\end{tabular}


Lesotho: Summary of the Tax System, February 14, 2001

(All amounts in maloti)

\begin{tabular}{llll}
\hline Tax & Nature of Tax & Exemptions and Deductions & Rates \\
\hline
\end{tabular}

4. Taxes on international trade and transactions

4.1 Customs duties

Customs and Excise Act, No. 10 of 1982.

Notice No. 71 of 1969 (effective March 1, 1970).

4.2 Livestock importation levy Legal Notice No. 196 of 1991.
A duty on all goods imported into Lesotho. A three-column tariff scheduled based on the Customs Cooperation Council (CCC) nomenclature with fiscal, general, and most-

favored nation (MFN) rates of duty used. Goods originating from countries enjoying MFN status pay the fiscal and

customs duties. There is no preferential rate of duty.

A duty collected by the Republic of South Africa and other partners (at port of arrival) and contributed to a common customs union pool held with Botswana, Namibia, South Africa, and Swaziland.

Lesotho's share is calculated according to a fixed formula which is presently being reviewed.

A duty collected by the

Ministry of Agriculture when the import permits are issued.
There are free-trade agreements with Botswana,

Namibia, South Africa, and Swaziland.
Ad valorem duties charged on the domestic value of the goods at varying rates.
For private persons: M 30 and
M 15 for each head of large and
small stock, respectively.
For licensed butchers: M 7.50
and
M 3.75 for each head of large 
Lesotho: Summary of the Tax System, February 14, 2001

(All amounts in maloti)

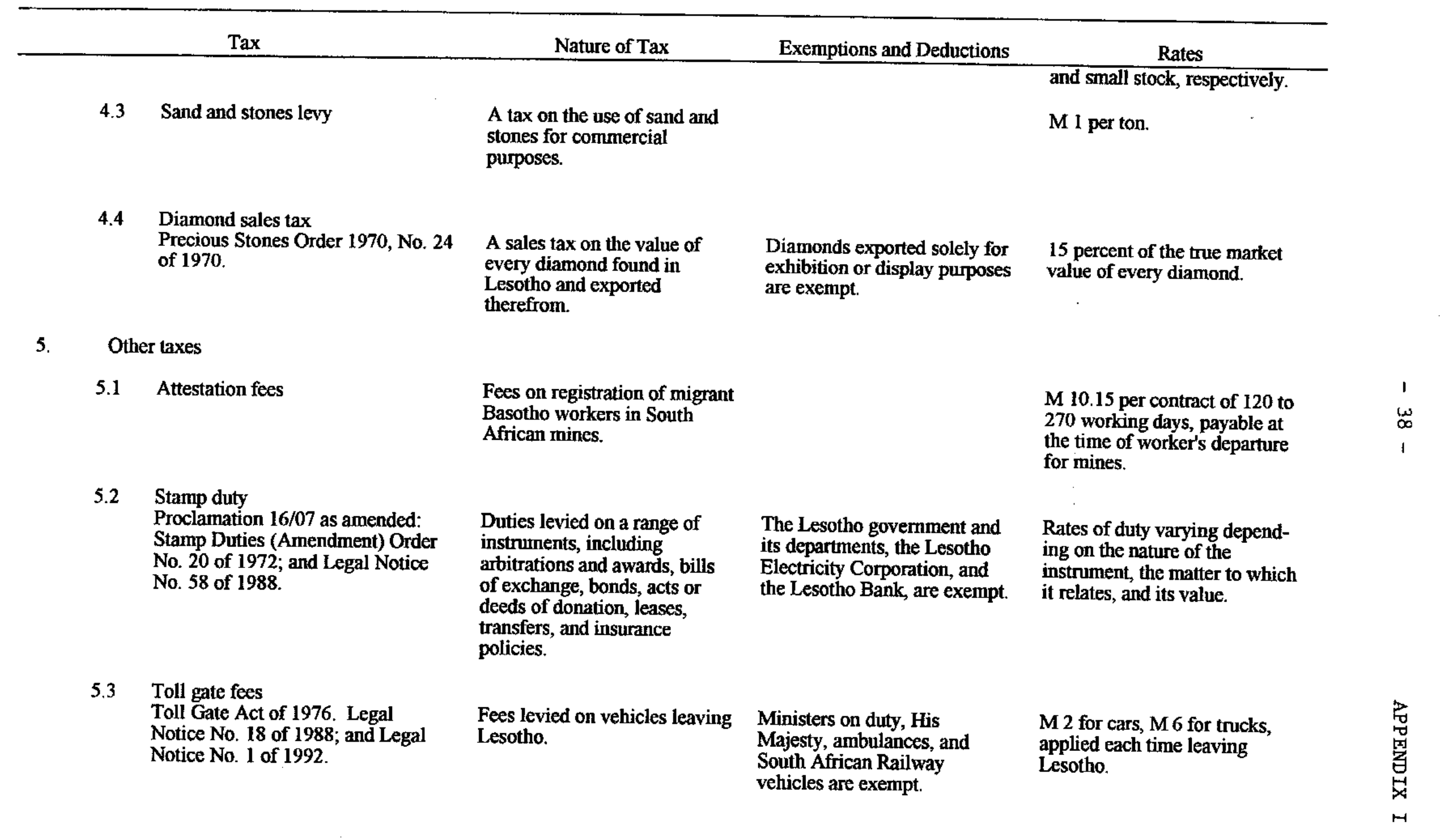


Lesotho: Summary of the Tax System, February 14, 2001

(All amounts in maloti)

\begin{tabular}{|c|c|c|c|c|}
\hline & $\operatorname{Tax}$ & Nature of Tax & Exemptions and Deductions & Rates \\
\hline 6. & $\begin{array}{l}\text { Pioneer Industries } \\
\text { Pioneer Industries Encouragement Act, } \\
1969, \text { No. } 19 \text { of } 1969 \text {, as amended. }\end{array}$ & $\begin{array}{l}\text { Applicable to manufacturers } \\
\text { and related industries and } \\
\text { building companies } \\
\text { establishing their operations in } \\
\text { Lesotho that had already been } \\
\text { approved for tax benefits to } \\
\text { encourage pioneering } \\
\text { industries by August } 3,1990 \text {. }\end{array}$ & $\begin{array}{l}\text { Approved manufacturers may } \\
\text { opt for ten years' exemption } \\
\text { from income tax, with five } \\
\text { more years carry-forward of } \\
\text { assessed losses or a package of } \\
\text { tax allowances, which can be } \\
\text { extended for approved } \\
\text { expansion An approved } \\
\text { existing manufacturer or a } \\
\text { hotel or casino-keeper is } \\
\text { limited to the package of } \\
\text { allowances. The incentives } \\
\text { can be revoked, varied, or } \\
\text { extended according to the } \\
\text { performance of the approved } \\
\text { manufacturer, with the } \\
\text { approval of the Minister of } \\
\text { Trade. }\end{array}$ & $\begin{array}{l}\text { Option for companies approved } \\
\text { for tax benefits (mentioned in } \\
\text { adjoining column) by August } 3 \text {, } \\
1990 \text { of surrendering such } \\
\text { benefits and immediately } \\
\text { switching over to the } 15 \text { percent } \\
\text { company tax rate or of retaining } \\
\text { these benefits and paying the } \\
\text { tax rate of } 35 \text { percent upon } \\
\text { expiry of existing tax holidays. } \\
\text { Most tax holiday companies } \\
\text { already exercised this option } \\
\text { even before the report of the } \\
\text { Pioneering Industries } \\
\text { Encouragement Act. }\end{array}$ \\
\hline
\end{tabular}

Source: Ministry of Finance. 


\section{Lesotho: Exchange and Trade System}

(Position as of December 31, 2000)

Subject Measure

Subject Measure

Date of membership

1. Article VIII

2. Article XIV
Existence

Description

\section{Status Under IMF Articles of Agreement}

July 25,1968 .

Yes Date of acceptance: March 5, 1997.

No

\section{Exchange Arrangements}

\section{Currency}

Other legal tender

\section{Exchange rate structure}

a. Unitary

b. Dual

c. Multiple

\section{Classification}

a. Exchange rate, no separate legal tender

b. Currency board arrangements

c. Conventional pegged arrangement

d. Pegged exchange rate within bands

e. Crawling peg

f. Crawling band

g. Managed floating, no preannounced path

h. Independently floating

\section{Exchange tax}

5. Exchange subsidy

6. Forward exchange market
Yes

Yes

Yes

n.a.

Yes The loti is pegged to the South African rand at $M 1$ per $R 1$.
No

No

Yes Authorized dealers are permitted to conduct forward exchange operations through their correspondent banks abroad at rates quoted by the latter. Forward exchange cover, however, is not common in Lesotho. 


\section{Lesotho: Exchange and Trade System}

(Position as of December 31, 2000)

\begin{tabular}{lll} 
Subject Measure & Existence & Description \\
\hline Official coverage & n.a.
\end{tabular}

\section{Arrangements for Payments and Receipts}

\section{Prescription of currency requirements Yes}

\section{Payments arrangements}

a. Bilateral payment agreement

(1) Operative

(2) Inoperative

b. Regional arrangement

c. Clearing agreement

d. Barter agreement and open accounts

3. Administration of control

Yes

No

No

No

Yes

No

No

Yes
Settlements by or to residents of the Common Monetary Area (CMA) with all countries outside the CMA may be made in rand to and from a nonresident account and in any foreign currency.

As Lesotho is part of the CMA, payments within the CMA are unrestricted and unrecorded except for statistical and customs purposes. In its relations with countries outside the CMA, Lesotho applies exchange controls that are largely similar to those applied by South Africa and Swaziland.
The Central Bank of Lesotho (CBL) controls foreign exchange transactions and delegates to commercial banks the authority to approve certain types of current payments up to established limits. Permits are issued by the Department of Customs and Excise based on the recommendation of the Department of Trade and Industry. Licenses for financial institutions accepting deposits and insurance companies, brokers, and agents are issued by the CBL.

\section{International security restrictions No}

a. In accordance with Executive Board decisio No No. 144(52/51)

b. According to UN Sanctions No

\section{Payment arrears No}

a. Official No

b. Private No

6. Controls on trade in gold (coins/bullions Yes 


\section{Lesotho: Exchange and Trade System}

(Position as of Decernber 31, 2000)

\begin{tabular}{|c|c|c|}
\hline Subject Measure & Existence & Description \\
\hline a. On domestic ownership/trade & & Only authorized dealers may trade in gold, but anyone may hold gold. \\
\hline b. On external trade & Yes & Exports of gold from the CMA are prohibited. \\
\hline 7. Controls on banknotes & Yes & \\
\hline a. On exports & Yes & \\
\hline (1) Domestic currency & Yes & Exports of currency from Lesotho are prohibited. \\
\hline (2) Foreign currency & Yes & $\begin{array}{l}\text { Exports of foreign currency from the CMA by residents are prohibited; } \\
\text { visitors may reexport the unspent portion of foreign currency brought } \\
\text { into the country. }\end{array}$ \\
\hline b. On imports & No & \\
\hline (1) Domestic currency & No & \\
\hline (2) Foreign currency & No & \\
\hline
\end{tabular}

\section{Resident Accounts}

1. Foreign exchange accounts permitted Yes

a. Held domestically

Approval required

b. Held abroad

Approval required

2. Accounts in domestic currency converti Yes in foreign currency

Yes

Yes

Yes

Yes
Banks may hold foreign exchange accounts abroad.

Only banks may hold these accounts.

Approval is required.

\section{Nonresident Accounts}

\section{Foreign exchange accounts permitted Yes}

Approval required

\section{Domestic currency accounts}

Convertible into foreign currency

Approval required

\section{Blocked accounts}

Yes

Yes

Yes

Yes

Yes
Loti accounts of nonresidents are divided into nonresident accounts and emigrant blocked accounts. 


\section{Lesotho: Exchange and Trade System}

(Position as of December 31, 2000)

\begin{tabular}{lc}
\multicolumn{1}{c}{ Subject Measure } & Exist \\
\hline & VL \\
\hline 1. Foreign exchange budget & No \\
2. Financing requirements for imports & Yes \\
a. Minimum financing requirements & No \\
b. Advance payments requirements & Yes
\end{tabular}

c. Advance import deposits

No

3. Documentation requirements for release foreign exchange for imports
a. Domiciliation requirements
Yes
b. Preshipment inspection
Yes
c. Letters of credit
Yes
d. Import licenses used as exchange licenses
e. Other
Yes
Yes

4.Import licenses and other nontariff Yes measures

Lesotho is a member of the Southern African Customs Union (SACU), and all imports, except certain food imports, originating in any country of the SACU are unrestricted. Imports from countries outside the SACU are usually licensed in conformity with the import regulations of the SACU. Lesotho reserves the right to restrict certain imports. Import permits are valid for all countries and entitle the holder to buy the foreign exchange required to make payments for imports from outside the SACU.
a. Positive list
No
b. Negative list
Yes

With certain exceptions, imports from outside the SACU must conform to a negative list and be licensed, (example, ammunition, flora and fauna, illegal drugs, etc.).

c. Open general licenses

No

d. Licenses with quotas

No

Certain food imports from within the SACU are subject to import licensing. 


\section{Lesotho: Exchange and Trade System}

(Position as of December 31, 2000)

\begin{tabular}{lll}
\hline \multicolumn{1}{c}{ Subject Measure } & Existence & Description \\
\hline e. Other nontariff measures & No & Lesotho applies the external customs tariffs of the SACU. \\
5. Import taxes/tariffs & Yes & \\
Taxes collected through the exchange system & No & \\
6. State import monopoly & No & \\
\hline
\end{tabular}

VII. Exports and Export Proceeds

\section{Repatriation requirements}

Surrender requirements

\section{Financing requirements}

\section{Documentation requirements}

a. Letter of credit

b. Guarantees

c. Domiciliation

d. Preshipment inspection

e. Other

4. Export licenses

a. Without quotas

b. With quotas

\section{Export taxes}

a. Collected through the exchange system

b. Other export taxes
Yes

Yes

Yes

Yes

Yes

Yes

Yes

Yes

No

Yes

Yes

No

No

No

No
All export proceeds must be repatriated within six months.

Unless otherwise permitted, all export proceeds must be surrendered to authorized dealers within six months of the date of the export transaction.

A state-supported export credit scheme is in effect, involving credit guarantees, and pre- and postshipment credits. 


\section{Lesotho: Exchange and Trade System}

(Position as of December 31, 2000)

\begin{tabular}{ll}
\multicolumn{1}{c}{ Subject Measure } & Existe \\
\hline a. Prior approval & No \\
b. Quantitative limits & No \\
c. Indicative limits/bona fide tests & No \\
2. Investment-related payments & Yes
\end{tabular}
a. Prior approval
Yes
b. Quantitative limits
Yes

c. Indicative limits/bona fide tests

n.a. There is no indicative limit or bona fide test for the payment of

\section{Payments for travel Yes}

a. Prior approval Yes

b. Quantitative limits Yes

c. Indicative limits/bona fide tests Yes

There is a limit of $M 100,000$ for adults and M 30,000 for children under
12 years of age, per calendar year.

4. Personal payments Yes

a. Prior approval Yes There is prior approval for payment of study abroad costs.

b. Quantitative limits Yes

Profit and dividend transfers are not restricted, provided the funds were not obtained through excessive use of local borrowing facilities. depreciation of direct investments. commissions. Emigrants are allowed to transfer through normal banking channels, up
to $\mathrm{M} 300,000$ of earnings on blocked assets.

es

Larger allowances may be obtained for business travel.

c. Indicative limits/bona fide tests

No

\section{Foreign workers' wages No}

$\begin{array}{ll}\text { a. Prior approval } & \text { No } \\ \text { b. Quantitative limits } & \text { No } \\ \text { c. Indicative limits/bona fide tests } & \text { No }\end{array}$

For studies abroad the limits are $M 80,000$ per annum for a single student or M 160,000 per annum for a student accompanied by a spouse who is not studying.

\section{Credit card use abroad No}

a. Prior approval No

b. Quantitative limits No 


\section{Lesotho: Exchange and Trade System}

(Position as of December 31, 2000)

\begin{tabular}{lll}
\hline \multicolumn{1}{c}{ Subject Measure } & Existence & Description \\
\hline c. Indicative limits/bona fide tests & No & \\
7. Other payments & No \\
a. Prior approval & No \\
b. Quantitative limits & No \\
c. Indicative limits/bona fide tests & No \\
\hline
\end{tabular}

IX. Proceeds from Invisible Transactions and Current Transfers

\section{Repatriation requirements Yes}

Surrender requirements Yes Proceeds must be surrendered within 30 days of the date of accrual, unless an exemption is obtained.

\section{Restrictions on use of funds No}

\section{Capital Transactions}

\section{Controls on capital and money market Yes} instruments

a. On capital market securities Yes

(1) Shares or other securities of a participatin Yes nature
(a) Purchase locally by nonresidents
Yes
(b) Sale or issue locally by nonresidents
Yes
(c) Purchase abroad by residents
Yes
(d) Sale or issue abroad by residents
Yes
(2) Bonds or other debt securities
n.a.
(a) Purchase locally by nonresidents
n.a.
(b) Sale or issue locally by nonresidents
n.a.
(c) Purchase abroad by residents
Yes
(d) Sale or issue abroad by residents
Yes
b. On money market instruments
Yes
(1) Purchase locally by nonresidents
No
(2) Sale or issue locally by nonresidents
No 


\section{Lesotho: Exchange and Trade System}

(Position as of December 31, 2000)

\begin{tabular}{|c|c|c|}
\hline Subject Measure & Existence & Description \\
\hline (3) Purchase abroad by residents & Yes & \\
\hline (4) Sale or issue abroad by residents & Yes & \\
\hline c. On collective investment securities & No & \\
\hline (1) Purchase locally by nonresidents & No & \\
\hline (2) Sale or issue locally by nonresidents & No & \\
\hline (3) Purchase abroad by residents & Yes & \\
\hline (4) Sale or issue abroad by residents & Yes & \\
\hline $\begin{array}{l}\text { 2. Controls on derivatives and other } \\
\text { instruments }\end{array}$ & No & \\
\hline a. Purchase locally by nonresidents & No & \\
\hline b. Sale or issue locally by nonresidents & No & \\
\hline c. Purchase abroad by residents & No & \\
\hline d. Sale or issue abroad by residents & No & \\
\hline 3. Controls on credit operations & Yes & \\
\hline a. Commercial credit & Yes & \\
\hline (1) By residents to nonresidents & Yes & $\begin{array}{l}\text { Export credits are available for up to six months; in certain } \\
\text { circumstances, the maturity can be extended by six months. Longer-term } \\
\text { credits require exchange control approval. }\end{array}$ \\
\hline (2) To residents from nonresidents & Yes & These credits require exchange control approval. \\
\hline b. Financial credit & Yes & \\
\hline (1) By residents to nonresidents & Yes & $\begin{array}{l}\text { These credits require prior approval. However, nonresident, wholly } \\
\text { owned subsidiaries may borrow locally up to } 100 \text { percent of the total } \\
\text { shareholder's investment. }\end{array}$ \\
\hline (2) To residents from nonresidents & Yes & $\begin{array}{l}\text { Prior approval is required to ensure that repayments and servicing of the } \\
\text { loans do not disrupt the balance of payments and to ensure that the level } \\
\text { of interest rates paid is reasonable in terms of prevailing international } \\
\text { rates. }\end{array}$ \\
\hline $\begin{array}{l}\text { c. Guarantees, sureties, and financial backup } \\
\text { securities }\end{array}$ & No & \\
\hline (1) By residents to nonresidents & No & \\
\hline
\end{tabular}




\section{Lesotho: Exchange and Trade System}

(Position as of December 31, 2000)

\begin{tabular}{|c|c|c|}
\hline Subject Measure & Existence & Description \\
\hline (2) To residents from nonresidents & No & \\
\hline 4. Controls on direct investment & Yes & $\begin{array}{l}\text { The rulings on applications for inward and outward capital transfers may } \\
\text { depend on whether the applicant is a temporary resident foreign national, } \\
\text { a nonresident, or a resident. }\end{array}$ \\
\hline a. Outward direct investment & Yes & Outward direct investment is prohibited. \\
\hline b. Inward direct investment & No & \\
\hline $\begin{array}{l}\text { 5. Controls on liquidation of direct } \\
\text { investment }\end{array}$ & No & \\
\hline 6. Controls on real estate transactions & Yes & \\
\hline a. Purchase abroad by residents & Yes & Prior approval is required. \\
\hline b. Purchase locally by nonresidents & No & \\
\hline c. Sales locally by nonresidents & No & \\
\hline 7. Controls on personal capital movements & No & \\
\hline a. Loans & No & \\
\hline (1) By residents to nonresidents & No & \\
\hline (2) To residents from nonresidents & No & \\
\hline b. Gifts, endowments, inheritances, and legaci & No & \\
\hline (1) By residents to nonresidents & No & \\
\hline (2) To residents from nonresidents & No & \\
\hline c. Settlements of debt abroad by immigrants & No & \\
\hline d. Transfer of assets & No & \\
\hline (1) Transfer abroad by emigrants & No & \\
\hline (2) Transfer into the country by immigrants & No & - \\
\hline e. Transfer of gambling/prize earnings & Yes & Prior approval is required. \\
\hline $\begin{array}{l}\text { 8. Specific controls on transactions by } \\
\text { commercial banks and other credit } \\
\text { institutions }\end{array}$ & Yes & \\
\hline a. Borrowing abroad & Yes & Prior approval is required. \\
\hline b. Maintenance of accounts abroad & Yes & \\
\hline
\end{tabular}




\section{Lesotho: Exchange and Trade System}

(Position as of December 31, 2000)

\begin{tabular}{ll}
\hline \multicolumn{1}{c}{ Subject Measure } & Exist \\
\hline $\begin{array}{l}\text { c. Lending to nonresidents (loans, financial or } \\
\text { commercial credit) }\end{array}$ & Yes \\
$\begin{array}{l}\text { d. Lending locally in foreign exchange to } \\
\text { residents }\end{array}$ \\
$\begin{array}{ll}\text { e. Purchase of locally issued securities } \\
\text { denominated in foreign exchange }\end{array}$ \\
$\begin{array}{ll}\text { f. Differential treatment of deposit accounts in } \\
\text { foreign exchange }\end{array}$ \\
$\begin{array}{ll}\text { (1) Reserve requirements } \\
\text { (2) Liquid asset requirements }\end{array}$ \\
$\begin{array}{ll}\text { (3) Interest rate controls } & \text { Yes } \\
\text { (4) Credit controls } & \text { Yes } \\
\end{array}$
\end{tabular}

g. Differential treatment of nonresident deposi No accounts or deposit accounts in foreign exchange
(1) Reserve requirements
n.a.
(2) Liquid asset requirements
n.a.
(3) Interest rate controls
No
(4) Credit controls
No
h. Investment regulations
n.a.
(1) Abroad by banks
No
(2) In banks by nonresidents
No

i. Open foreign exchange position limits

n.a.

(1) On nonresident assets and liabilities

n.a.

(2) On resident assets and liabilities

n.a.

\section{Provisions specific to institutional} investors

n.a.

a. Limits (max.) on securities issued by nonresidents and on portfolio invested abroad

b. Limits (max.) on portfolio invested abroad

n.a.

Authorized dealers may lend up to $\mathrm{M} 20,000$, provided that the total available to the borrower from any source does not exceed this amount. Facilities in excess of such an amount need prior approval. Yes

Yes

Yes

es

No

a.

.




\section{Lesotho: Exchange and Trade System}

(Position as of December 31, 2000)

\begin{tabular}{|c|c|c|}
\hline Subject Measure & Existence & Description \\
\hline c. Limits (min.) on portfolio invested locally & n.a. & \\
\hline $\begin{array}{l}\text { d. Currently matching regulations on } \\
\text { assets/liabilities composition }\end{array}$ & n.a. & \\
\hline $\begin{array}{l}\text { 10. Other controls imposed by securities } \\
\text { laws }\end{array}$ & n.a. & \\
\hline
\end{tabular}

